\title{
A History and Survey of the Novikov Conjecture
}

\author{
Steven C. Ferry, Andrew Ranicki, and \\ Jonathan Rosenberg
}

\section{Contents}

1. Precursors of the Novikov Conjecture 8

$\begin{array}{ll}\text { Characteristic classes } & 8\end{array}$

Geometric rigidity $\quad 9$

The Hirzebruch signature theorem 9

The converse of the signature theorem (Browder, Novikov) 10

Topological invariance of the rational Pontrjagin classes (Novikov) 11

Non-simply-connected surgery theory (Novikov, Wall) 11

Higher signatures 12

Discovery of special cases of the Novikov Conjecture (Rokhlin, Novikov) 13

2. The Original Statement of the Novikov Conjecture 13

О нерешенных задачах 13

[An English Version:] Unsolved Problems 15

3. Work related to the Novikov Conjecture:

The First 12 Years or So 17

$\begin{array}{ll}\text { Statements of the Novikov and Borel Conjectures } & 17\end{array}$

$\begin{array}{ll}\text { Mishchenko and the symmetric signature } & 18\end{array}$

$\begin{array}{ll}\text { Lusztig and the analytic approach } & 20\end{array}$

Splitting theorems for polynomial extensions 21

Cappell and codimension 1 splitting theorems 22

Mishchenko and Fredholm representations 23

Farrell-Hsiang and the geometric topology approach 24

Kasparov and operator-theoretic $K$-homology 25

Surgery spectra and assembly (Quinn) 25

4. Work related to the Novikov Conjecture:

The Last 12 Years or So, I:

S. C. Ferry was partially supported by NSF Grant \# DMS-93-05758.

J. Rosenberg was partially supported by NSF Grant \# DMS-92-25063. 
Homotopy Theory and Algebra 27

Algebraic surgery theory (Ranicki) 27

The homotopy-limit problem, descent (Carlsson) 28

The Carlsson-Pedersen approach 30

Controlled, continuously controlled and bounded topology 30

$K$-theoretic analogues of the Novikov and Borel Conjectures 31

5. Work related to the Novikov Conjecture:

The Last 12 Years or So, II:

Geometric Topology 34

Farrell-Hsiang, Ferry-Weinberger and tangentiality 34

The Farrell-Jones program 37

Problems about group actions (Weinberger et al.) 39

6. Work related to the Novikov Conjecture:

The Last 12 Years or So, III:

Elliptic Operators and Operator Algebras 41

Further development of Kasparov $K K$-theory 41

The cyclic homology approach (Connes et al.) 42

$K$-theory of group $C^{*}$-algebras: the Connes-Kasparov and Baum-Connes Conjectures 44

Parallels with positive scalar curvature

(Gromov-Lawson, Rosenberg et al.) 45

Analogues for foliations 46

Flat and almost flat bundles revisited

(Connes-Gromov-Moscovici, Gromov et al.) 47

Index theory on non-compact manifolds (Roe et al.) 48

References $\quad 50$

\section{Precursors of the Novikov Conjecture}

\section{Characteristic classes}

The Novikov Conjecture has to do with the question of the relationship of the characteristic classes of manifolds to the underlying bordism and homotopy theory. For smooth manifolds, the characteristic classes are by definition the characteristic classes of the tangent (or normal) bundle, so basic to this question is another more fundamental one: how much of a vector bundle is determined by its underlying spherical fibration? The Stiefel-Whitney classes of vector bundles are invariants of the underlying spherical fibration, and so the Stiefel-Whitney numbers of manifolds are homotopy invariants. Furthermore, they determine unoriented bordism. The Pontrjagin classes of 
vector bundles are not invariants of the underlying spherical fibration, and the Pontrjagin numbers of manifolds are not homotopy invariants. However, together with the Stiefel-Whitney numbers, they do determine oriented bordism. The essential connection between characteristic numbers and bordism was established by Thom [Th1] in the early 1950's.

\section{Geometric rigidity}

As we shall see later, the Novikov Conjecture is also closely linked to problems about rigidity of aspherical manifolds. As everyone learns in a first course in geometric topology, closed 2-manifolds are determined up to homeomorphism by their fundamental groups. In higher dimensions, of course, nothing like this is true in general, but one can still ask if aspherical closed manifolds (closed manifolds having contractible universal cover) are determined up to homeomorphism by their fundamental groups. That this should be the case is the Borel Conjecture formulated by Armand Borel in the 50's (according to Hsiang in [Hs3]), and communicated to various people in the 60 's. In dimension 2, restricting attention to aspherical manifolds is little loss of generality, since $S^{2}$ and $\mathbb{R P}^{2}$ are the only closed 2-manifolds which are not aspherical. The Mostow Rigidity Theorem was the most dramatic early evidence for the Borel Conjecture, proving the conjecture for closed manifolds which are locally symmetric spaces.

\section{The Hirzebruch signature theorem}

The actual history of the Novikov Conjecture starts with the Hirzebruch signature theorem [Hir], which expresses the signature of an oriented closed $4 k$-dimensional manifold $M$ in terms of characteristic classes:

$$
\operatorname{signature}(M)=\langle\mathcal{L}(M),[M]\rangle \in \mathbb{Z}
$$

Here, $\mathcal{L}(M) \in H^{4 *}(M ; \mathbb{Q})$ is the $\mathcal{L}$-class of $M$, a certain formal power series in the Pontrjagin classes $p_{*}(M) \in H^{4 *}(M)$ with rational coefficients. The formula is surprising in that the left hand side is an integer which only depends on the structure of the cohomology ring of $M$, whereas the right hand side is a sum of rational numbers which are defined (at least $a$ priori) in terms of the differentiable structure. The (inhomogeneous) class $\mathcal{L}(M)$ determines all of the rational Pontrjagin classes of $M$, but only the component of $\mathcal{L}(M)$ in the dimension of $M$ is homotopy invariant - in fact, the other components are not even bordism invariants. Milnor [Miln1] used the signature theorem to verify that the homotopy spheres he constructed do indeed have exotic differentiable structures. 


\section{The converse of the signature theorem (Browder, Novikov)}

Following the development of Thom's bordism theory, Milnor [Miln2] proved that two manifolds are bordant if and only if they are related by a finite sequence of surgeries. This was the beginning of the use of surgery as a fundamental tool in differential topology. Soon afterwards, Kervaire and Milnor $[\mathrm{KerM}]$ used surgery to classify exotic spheres in dimensions $\geq 7$. In 1962, Browder ([Br1],[Br4]) and Novikov [Nov1], working independently, applied the same technique to manifolds with more complicated homology. They used surgery theory to establish a converse to the Hirzebruch signature theorem in dimensions $\geq 5$ : if $X$ is a simply-connected $4 k$-dimensional Poincaré space, such that the signature of $X$ is the evaluation on the fundamental class $[X] \in H_{4 k}(X)$ of $\mathcal{L}(-\nu)$ for some vector bundle $\nu$ with spherical Thom class, then $X$ is homotopy equivalent to a smooth closed manifold $M$ with stable normal bundle pulled back from $\nu$. A consequence of this is that for simply-connected $4 k$-dimensional manifolds in high dimensions, the top degree term of the $\mathcal{L}$-class is essentially the only homotopy-invariant rational characteristic class. Novikov ([Nov1], [Nov2], [Nov3]) extended these ideas to the study of the uniqueness properties of manifold structures within a homotopy type. Sullivan [Sul] then combined the Browder-Novikov surgery theory with homotopy theory to reformulate the surgery classification of manifolds in terms of the surgery exact sequence of pointed sets, which for a $4 k$-dimensional simply-connected manifold $M(k>1)$ has the form:

$$
0 \rightarrow \mathcal{S}(M) \stackrel{\theta}{\rightarrow}[M, G / O] \stackrel{A}{\rightarrow} \mathbb{Z}
$$

with $\mathcal{S}(M)$ the structure set of $M$, consisting of the equivalence classes of pairs $(N, f)$ with $N$ a closed manifold and $f: N \rightarrow M$ a homotopy equivalence. Two such pairs $(N, f),\left(N^{\prime}, f^{\prime}\right)$ are equivalent if there exists a diffeomorphism $g: N \rightarrow N^{\prime}$ with a homotopy $f^{\prime} g \simeq f: N \rightarrow M$. Here $G / O$ is the homotopy fiber of the forgetful map $J$ from the classifying space $B O$ for stable vector bundles to the classifying space $B G$ for stable spherical fibrations, and the map $\theta$ sends an element $(N, f)$ of the structure set to the difference between the stable normal bundle of $M$ and the push-forward under $f$ of the stable normal bundle of $N$. (Both are lifts of the same underlying spherical fibration, the Spivak normal fibration [Spv].) The map $A$ sends an element of $[M, G / O]$, represented by a vector bundle $\eta$ over $M$ with a fiber homotopy trivialization, to

$$
\left\langle\mathcal{L}\left(\tau_{M} \oplus \eta\right)-\mathcal{L}\left(\tau_{M}\right),[M]\right\rangle
$$

where $\tau_{M}$ is the tangent bundle of $M$. 


\section{Topological invariance of the rational Pontrjagin classes (Novikov)}

Around 1957, Thom [Th2] and Rokhlin and Shvarts [RokS], working independently, proved that the rational Pontrjagin classes of $P L$ manifolds are combinatorial invariants. As we have just explained, the work of Browder showed that the Pontrjagin classes are very far from being homotopy invariants of closed differentiable manifolds. Nevertheless, Novikov in 1966 ([Nov4], [Nov5], [Nov6]) was able to prove a most remarkable fact: the rational Pontrjagin classes are topological invariants. An essential feature of the proof was the use of non-simply-connected compact manifolds with free abelian fundamental group (e.g., tori), and of their non-compact universal covers.

\section{Non-simply-connected surgery theory (Novikov, Wall)}

While the basic methods used in Browder-Novikov surgery theory make sense without assuming simple connectivity, it was soon realized that formulating the correct results in the non-simply connected case is not so easy. For one thing, correctly understanding Poincaré duality in this context requires using homology with local coefficients. In fact, the correct algebraic approach required developing a theory of quadratic forms defined over an arbitrary ring with involution, the prototype being the integral group ring $\mathbb{Z}[\pi]$ of the fundamental group $\pi$. This algebra was developed in the evendimensional case by Novikov ([Nov8], [Nov10]) and Wall, working independently, and in the odd-dimensional case by Wall [Wall1]. Using this algebra, Wall [Wall2] developed a non-simply connected version of the surgery exact sequence for a closed $n$-dimensional manifold $M$ with $n \geq 5$ :

$$
\cdots \rightarrow L_{n+1}\left(\mathbb{Z}\left[\pi_{1}(M)\right]\right) \rightarrow \mathcal{S}(M) \stackrel{\theta}{\rightarrow}[M, G / O] \stackrel{A}{\rightarrow} L_{n}\left(\mathbb{Z}\left[\pi_{1}(M)\right]\right)
$$

The $L$-groups are Witt groups of $(-)^{k}$-quadratic forms on finitely generated free modules over the group ring for even $n=2 k$, and stable automorphism groups of such forms for odd $n=2 k+1$. The $L$-groups are periodic in $n$, with period 4 . While $\mathcal{S}(M)$ is only a pointed set, not a group, it has an affine structure: $L_{n+1}\left(\mathbb{Z}\left[\pi_{1}(M)\right]\right)$ acts on $\mathcal{S}(M)$, and two elements with the same image under $\theta$ lie in the same orbit. Rationally, the map $\theta: \mathcal{S}(M) \rightarrow$ $[M, G / O]$ sends a homotopy equivalence $f: N \rightarrow M$ to the difference $f_{*}(\mathcal{L}(N))-\mathcal{L}(M)$. Here the push-forward map $f_{*}$ can be defined as $g^{*}$, where $g$ is a homotopy inverse to $f$.

Shortly after the work of Wall, new advances by Kirby and Siebenmann made it possible to carry surgery theory over from the category of differentiable manifolds to the category of topological manifolds [KirS]. One again obtained a surgery exact sequence of the same form as before, but with 
$G / O$ replaced by $G / T O P$. This theory made it possible to reinterpret Novikov's theorem on the topological invariance of rational Pontrjagin classes as the fact that the forgetful map $G / T O P \rightarrow G / O$ induces an isomorphism on rational homology. The classifying spaces $G / O$ and $G / T O P$ both have rational cohomology rings which are formal power series algebras in the Pontrjagin classes.

\section{Higher signatures}

Let $\Gamma$ be a discrete group. A rational cohomology class $x \in H^{*}(B \Gamma ; \mathbb{Q})$ may be interpreted as a characteristic class for manifolds with fundamental group $\Gamma$. If $\Gamma=\pi_{1}(M)$ for a manifold $M$, obstruction theory implies that one can always find a map $u: M \rightarrow B \Gamma$ which induces an isomorphism on $\pi_{1}$. For oriented $M$ the class $x$ defines a (rational) characteristic number, called a higher signature:

$$
\operatorname{signature}_{x}(M, u)=\left\langle\mathcal{L}(M) \cup u^{*}(x),[M]\right\rangle \in \mathbb{Q} .
$$

This characteristic number is said to be homotopy invariant if for all orientation-preserving homotopy equivalences $f: N \rightarrow M$ of closed oriented manifolds and all maps $u: M \rightarrow B \Gamma$,

$$
\operatorname{signature}_{x}(M, u)=\operatorname{signature}_{x}(N, u \circ f) \in \mathbb{Q} .
$$

It is now possible to determine when this is the case. Because of the $L$ groups in the surgery sequence, there can be far more homotopy-invariant characteristic classes than in the simply connected case. Let $\theta_{(M, u)}^{*}$ be the map sending $x \in H^{*}(B \Gamma ; \mathbb{Q})$ to the functional on structure sets sending

$$
N \stackrel{f}{\rightarrow} M \stackrel{u}{\rightarrow} B \Gamma
$$

to

$$
\operatorname{signature}_{x}(M, u)-\operatorname{signature}_{x}(N, u \circ f) .
$$

By definition, the homotopy-invariant higher signatures are exactly those signature $_{x}$ 's for which $x$ is in the kernel of $\theta_{(M, u)}^{*}$, for all $M$ and $u$. The surgery exact sequence shows that these are precisely the $x$ 's in the image of a certain map

$$
A^{*}: \operatorname{Hom}\left(L_{*}(\mathbb{Z}[\Gamma]), \mathbb{Q}\right) \rightarrow H^{*}(B \Gamma ; \mathbb{Q})
$$

The Novikov Conjecture is that every higher signature is homotopy-invariant, or equivalently that $A^{*}$ is onto, for every discrete group $\Gamma$. 


\section{Discovery of special cases of the Novikov Conjecture (Rokhlin, Novikov)}

Novikov's use of manifolds with free abelian fundamental group in the proof of the topological invariance of rational Pontrjagin classes led him to the study of homotopy invariance properties of other characteristic classes as well. In particular, he studied the mod- $p$ Pontrjagin classes of homotopy lens spaces ([Nov7], [Nov9]), and the higher signatures of general non-simply connected manifolds. Novikov himself discovered that the higher signature (in this case there is essentially only one) of a manifold with infinite cyclic fundamental group is a homotopy invariant [Nov7], and Rokhlin [Rokh] studied the case of $\Gamma=\mathbb{Z} \times \mathbb{Z}$. These examples led Novikov to the formulation of the general conjecture.

\section{The Original Statement of the Novikov Conjecture}

The statement that is now usually known as the Novikov Conjecture first appears in complete form in $\S 11$ of S. P. Novikov's monumental paper [Nov10]. A slightly different formulation was given in Novikov's talk at the International Congress in Nice in 1970 [Nov8]. More preliminary versions had appeared in the lectures of Novikov for the de Rham Festschrift [Nov9] and the Moscow International Congress [Nov7]. Since the name "Novikov Conjecture" these days seems to mean quite different things to different people, in the interests of historical accuracy, we quote here the complete text of Novikov's original (Izvestia) formulation, both in the original Russian and in an English translation. As we shall see shortly, Novikov's original formulation already includes the three main approaches to the conjecture: the analytic, the topological, and the algebraic. Here is first the original Russian (with a few misprints corrected) and then a translation (our own correction of the printed translation in [Nov10]). The footnote indexed ${ }^{*}$ is Novikov's; numbered footnotes in the English version are ours.

\section{О нерешенных задачах}

1. Здесь мы обсудим первоначально следующий общий вопрос: что такое "общая неодносвязная Формула Хирцебруха"?

На этот вопрос можно ответить таким образом: должен существовать некоторый гомоморфизм "обобщенных сигнатур"

$$
\sigma_{k}: U_{1}^{n}(A) \rightarrow H_{n-4 k}\left(\pi_{1} ; \mathbb{Q}\right)
$$

такой, что для любого $n$-мерного замкнутого ориентированного многообразия $M^{n}$ с фундаментальной группой $\pi_{1}$ и естественного ото- 
бражения $f: M^{n} \rightarrow K\left(\pi_{1}, 1\right)$ скалярное произведение

$$
\left\langle L_{k}\left(M^{n}\right), D f^{*}(x)\right\rangle
$$

гомотопически инвариантно при всех $x \in H^{*}\left(\pi_{1} ; \mathbb{Q}\right)$, и $D L_{k}$ как линейная форма на $H^{*}\left(\pi_{1}\right)$-или элемент $H_{*}\left(\pi_{1} ; \mathbb{Q}\right)$ 一принадлежит образцу $\sigma_{k}$. Мы явно построили такие гомоморфизмы для одной абелевой группы-они оказались здесь даже изоморфизмами над $\mathbb{Q}$ (неэффективно это было известно в топологии-см. [HsS], [Sh1], [Wall1]).

Конечно, эта задача может быть поставлена и для конечных модулей $p$-по крайней мере для больших $p$ сравнительно с $n$.

Заметим, что ряд соображений подсказывает, что, например, для фундаментальных групп "солв" и “ниль"-многообразий такого рода гомоморфизм существует и является эпиморфизмом над $\mathbb{Q}$, так что допустимые классы циклов-это не только пересечение циклов коразмерности 1. Здесь можно ввести "некоммутативное расширение" кольца $A$-прибавление $z, z^{-1}$ без коммутирования с $A$-обобщить теорню операторов типа Басса. Однако общего вопроса это не проясняет. Разумеется, более прост вопрос об "относительных Формулах Хирцебруха". Отметим, что существенно более сложным является вопрос о внутреннем вычислении скалярных произведений $L_{k}$ с циклами вида $D f^{*}(x)$ даже для абелевых $\pi$-он не решен уже для $\pi=\mathbb{Z} \times \mathbb{Z}($ см. [Nov6], [Nov7], [Rokh]).*

2. Посмотрим, во что переходит вопрос о "неодносвязной формуле Хирцебруха" и построении гомоморфизмов "обобщенных сигнатур"

$$
\sigma: U_{1}^{*}(A) \rightarrow H^{*}(\pi ; \mathbb{Q})
$$

при замене групповых колец $A$ кольцами функций $A=C(X)$.

Если заменить $H_{*}(\pi ; \mathbb{Q})$ на $H^{*}(X)$, то мы ириходим к кадаче об абстрактно алгебраическом построении характера Черна

$$
\text { Ch }: U^{*}(A)=K^{*}(X) \rightarrow H^{*}(X) \text {. }
$$

При этом надо исходить из каого-то чисто кольцевого алгебраического формализма в построении $H^{*}(X)$-от кольца $C(X)$.

*А. С. Мищенко нашӭл своеобразный аналог классической сигнатуры-многообразию гомотопически инвариантным способом сопоставляется элемент из $U^{*}\left(\pi_{1}\right) \otimes \mathbb{Z}\left[\frac{1}{2}\right]$, что определяет гомоморфизм теории бордизмов $\Omega_{*}^{S O}\left(\pi_{1}\right) \rightarrow U^{*}\left(\pi_{1}\right) \otimes \mathbb{Z}\left[\frac{1}{2}\right]$ в эрмитову $K$-теорию, связанный, вероятно, с $L$-родом. 


\section{[An English Version:] Unsolved Problems}

1. Here we consider first of all the following question: what should be the "general non-simply connected Hirzebruch formula"?

The question can be answered as follows: there should exist a certain "generalized signature" ${ }^{1}$ homomorphism

$$
\sigma_{k}: U_{1}^{n}(A) \rightarrow H_{n-4 k}\left(\pi_{1} ; \mathbb{Q}\right) \quad 2
$$

such that for any $n$-dimensional closed oriented manifold $M^{n}$ with fundamental group $\pi_{1}$ and for the natural map ${ }^{3} f: M^{n} \rightarrow K\left(\pi_{1}, 1\right)$, the scalar product $\left\langle L_{k}\left(M^{n}\right), D f^{*}(x)\right\rangle^{4}$ is homotopy-invariant for any $x \in H^{*}\left(\pi_{1} ; \mathbb{Q}\right)$; and $D L_{k}$ as a linear form on $H^{*}\left(\pi_{1}\right)$ - or regarded as an element of $H_{*}\left(\pi_{1}\right.$, Q) ${ }^{5}$-belongs to the image of $\sigma_{k}$. We have explicitly constructed such homomorphisms for one class of abelian groups (viz., free abelian groups) - they turn out to be isomorphisms over $\mathbb{Q}$ (this was known non-effectively from results in topology - cf. [HsS], [Sh1], [Wall1]).

Of course, this problem can be posed for a finite modulus $p$, at least for $p$ large compared with $n .^{6}$

Let us note that a number of considerations suggest that, for example, for the fundamental groups of "solv-" and "nil-" manifolds, such a homomorphism exists and is an epimorphism over $\mathbb{Q}$, such that the allowable homology classes are not just the intersections of cycles of codimension 1. Here we can introduce a "non-commutative extension" of the ring $A$, by adjoining $z$ and $z^{-1}$ without assuming that they commute with $A$, to gen-

\footnotetext{
${ }^{1}$ In modern language, perhaps "higher signature" would be more appropriate.

${ }^{2}$ Here $A=\mathbb{Z}\left[\pi_{1}\right]$ is the group ring, and $U_{1}^{n}(A)$ is a certain variant of the Wall group $L_{n}(A)$; the exact decoration on the surgery group is unimportant since we are ignoring torsion here anyway. The homomorphisms $\sigma_{*}$ are exactly what one needs to have a rational splitting of the $L$-theory assembly map.

3 the classifying map for the universal cover of $M$

${ }^{4}$ Here $L_{k}$ is the component of the total Hirzebruch $L$-class in degree $4 k$, and $D$ denotes the Poincaré dual, or $\cap$-product with the fundamental class $[M]$ determined by the orientation.

${ }^{5}$ meaning $f_{*}\left(D L_{k}\left(M^{n}\right)\right)$

${ }^{6}$ It seems that here Novikov is referring back to a problem discussed in $\S 3$ of his paper [Nov9], concerning topological and homotopy invariance of "mod- $p$ " Pontrjagin classes. While the mod- $p$ Pontrjagin classes are in general not even homeomorphism invariants, Corollary $\mathrm{C}$ in [Nov9, §3] asserts that for any integer $n \geq 2$, the tangential homotopy type of lens spaces obtained as quotients of $S^{2 n-1}$ by linear representations of $\mathbb{Z} / p$ on $\mathbb{C}^{n}$ (free away from the origin) is a topological invariant, provided that $p$ is sufficiently large compared with $n$.
} 
eralize the theory of operators of Bass type. ${ }^{7}$ However, this does not clarify the general question. It goes without saying that the question of a "relative Hirzebruch formula" is simpler. ${ }^{8}$ Let us note that the question of the intrinsic calculation of scalar products of $L_{k}$ with cycles of the form $D f^{*}(x)$ is essentially more complicated even for abelian $\pi$-it has not been solved even for $\pi=\mathbb{Z} \times \mathbb{Z}$ (see [Nov6], [Nov7], [Rokh]). ${ }^{* 9}$

2. Let us see what the question about a "general non-simply connected Hirzebruch formula" and the construction of "generalized signature" homomorphisms

$$
\sigma_{k}: U_{1}^{*}(A) \rightarrow H_{*}(\pi ; \mathbb{Q})
$$

becomes when we replace the group ring $A$ by a ring of functions $A=$ $C(X) \cdot{ }^{10}$

If we replace $H_{*}(\pi ; \mathbb{Q})$ by $H^{*}(X)$ then we arrive at a problem about the abstract algebraic construction of the Chern character

$$
\text { Ch }: U^{*}(A)=K^{*}(X) \rightarrow H^{*}(X) \text {. }
$$

For this it is necessary to start from some purely ring-theoretic formalism for constructing $H^{*}(X)$ from the ring $C(X) .{ }^{11}$

\footnotetext{
${ }^{7}$ Without saying so, Novikov is sketching here an inductive method of proving the Novikov Conjecture for poly- $\mathbb{Z}$ groups in a purely algebraic way. For the free abelian case, one needs an analogue in $L$-theory of the Bass-Heller-Swan decomposition of the $K$-theory of a Laurent polynomial ring $A\left[z, z^{-1}\right]$ ([BHS]). This was first provided in work of Shaneson [Sh2]. A similar method will work for poly- $\mathbb{Z}$ groups but it is necessary to work with twisted Laurent rings or crossed products

$$
A \rtimes_{\alpha} \mathbb{Z}=A_{\alpha}\left[z, z^{-1}\right]:=\left\langle A, z, z^{-1} \mid z a z^{-1}=\alpha(a), \quad a \in A\right\rangle
$$

and to prove a "Bass type" theorem for those. Such theorems for twisted Laurent rings were later provided by Farrell and Hsiang ([FarHs2], [FarHs3]) for algebraic $K$-theory, by Cappell [Cap2] and Ranicki [Ran3] for algebraic $L$-theory, and by Pimsner-Voiculescu $[\mathrm{PimV}]$ for the $K$-theory of $C^{*}$-algebras. Specific applications to the Novikov Conjecture were provided in [FarHs4], [FarHs5] and in [Ros2], [Ros4].

${ }^{8}$ Novikov has pointed out to us that he was referring here to (relative) invariants of degree-one normal maps, which are easier to define than (absolute) invariants for closed manifolds.

*A. S. Mishchenko has found an analogue of the classical signature-a homotopyinvariant element of $U^{*}\left(\pi_{1}\right) \otimes \mathbb{Z}\left[\frac{1}{2}\right]$ associated to a manifold, which defines a homomorphism from bordism theory $\Omega_{*}^{S O}\left(\pi_{1}\right) \rightarrow U^{*}\left(\pi_{1}\right) \otimes \mathbb{Z}\left[\frac{1}{2}\right]$ to hermitian $K$-theory, related, apparently, to the $L$-genus.

${ }^{9}$ This is the symmetric signature of Mishchenko [Mis1] and Ranicki ([Ran4], [Ran5]).

${ }^{10}$ Here Novikov is anticipating what later became a major industry, of studying the Novikov Conjecture in the context of $C^{*}$-algebras rather than group rings. A ring of the form $C(X)$ is exactly the most general commutative (complex) $C^{*}$-algebra.

${ }^{11}$ This is of course exactly what Connes has done with the introduction of cyclic
} 


\section{Work related to the Novikov Conjecture: The First 12 Years or So}

\section{Statements of the Novikov and Borel Conjectures}

Let $\Gamma$ be a discrete group.

Novikov Conjecture for $\Gamma$. The higher signatures determined by $\Gamma$ are all homotopy invariant, i.e. for every rational cohomology class $x \in H^{*}(B \Gamma ; \mathbb{Q})$, for every orientation-preserving homotopy equivalence $f: N \rightarrow M$ of closed oriented manifolds and for every map $u: M \rightarrow B \Gamma$

$$
\text { signature }_{x}(M, u)=\text { signature }_{x}(N, u \circ f) \in \mathbb{Q} .
$$

Borel Conjecture for $\Gamma$. Every homotopy equivalence $f: N \rightarrow M$ of closed aspherical manifolds with $\pi_{1}(M)=\Gamma$ is homotopic to a homeomorphism. More generally, if $f:(N, \partial N) \rightarrow(M, \partial M)$ is a homotopy equivalence of compact manifolds with boundary such that $M$ is aspherical, $\pi_{1}(M)=\Gamma$ and $\partial f: \partial N \rightarrow \partial M$ is a homeomorphism, then $f$ is homotopic rel boundary to a homeomorphism.

The first part of the Borel Conjecture only applies to discrete groups $\Gamma$ such that the classifying space $B \Gamma$ is realized by a closed aspherical manifold $M$ with $\pi_{1}(M)=\Gamma, \pi_{i}(M)=0$ for $i \geq 2$. The more general part applies to any discrete group $\Gamma$ such that $B \Gamma$ is realized by a finite aspherical polyhedron $K$, since then any regular neighbourhood of $K$ in a high-dimensional Euclidean space is a compact manifold with boundary $(M, \partial M)$ such that $M \simeq K \simeq B \Gamma$ is aspherical. Such $\Gamma$ are finitely presented, but in a later section we shall also formulate a version of the Borel Conjecture for noncompact manifolds, which applies to $\Gamma$ which need not be finitely generated.

The Novikov and Borel Conjectures are only interesting for infinite groups $\Gamma$.

The $h$-cobordism version of the Borel Conjecture has the same hypothesis, but it is only required that the homotopy equivalence be $h$-cobordant to a homeomorphism. There is also an s-cobordism version of the Borel Conjecture in which it is required that the homotopy equivalence be simple: by the $s$-cobordism theorem for dimensions $\geq 6$ there exists a homotopy to a

homology, though one complication that Novikov seems not to have anticipated is the need to make a good choice of a dense subalgebra $\mathcal{A}$ of the $C^{*}$-algebra $A=C(X)$, which on the one hand has the property that the inclusion $\mathcal{A} \hookrightarrow A$ induces an isomorphism on (topological) $K$-theory, and on the other hand gives the correct cyclic homology groups. 
homeomorphism if and only if there exists an $s$-cobordism to a homeomorphism. The $h$ - and $s$-cobordism versions of the Borel Conjecture only differ from the actual Borel Conjecture in Whitehead torsion considerations. In particular, if $\mathrm{Wh}(\Gamma)=0$ the three versions of the conjecture coincide.

Surgery theory shows that the Borel Conjecture for $\Gamma$ implies the Novikov Conjecture for $\Gamma$, and that in fact the Borel Conjecture is an integral version of the Novikov Conjecture.

Also at about the same that Novikov's Izvestia paper appeared in print, Wall's monumental book [Wall2] appeared, giving for the first time a complete published account of the theory of non-simply connected surgery. The appendices to this book, written later than the main body of the text, contain Wall's slight reformulation of the Novikov Conjecture. Using Mishchenko's work on the symmetric signature (which is described in the next section) Wall made the first study of what is now :

Integral Novikov Conjecture for $\Gamma$. The assembly map in quadratic L-theory

$$
A_{\Gamma}: H_{*}\left(B \Gamma ; \mathbb{L}_{\bullet}(\mathbb{Z})\right) \rightarrow L_{*}(\mathbb{Z}[\Gamma])
$$

is an isomorphism for a torsion-free group $\Gamma$.

See the section below on surgery spectra for an account of the quadratic $L$-theory assembly map.

For a group $\Gamma$ which is the fundamental group of an aspherical manifold $M \simeq B \Gamma$ and is such that the Whitehead group of $\Gamma$ vanishes the Integral Novikov Conjecture is in fact equivalent to the Borel Conjecture in dimensions $\geq 5$.

\section{Mishchenko and the symmetric signature}

As Novikov indicated in a footnote (marked above with an asterisk) to his Izvestia paper, a useful technical tool, the symmetric signature, was developed by Mishchenko ([Mis1], [Mis2]) shortly after Novikov was led to the first version of his conjecture. Mishchenko worked not with quadratic forms over the integral group ring $\mathbb{Z}[\Gamma]$ of the fundamental group $\Gamma$, but rather with symmetric forms over the rational group ring $\mathbb{Q}[\Gamma]$ (though for rings containing $\frac{1}{2}$ there is no essential difference between quadratic and symmetric forms), and more generally with chain complexes $C$ over an arbitrary ring with involution $A$, with a symmetric Poincaré duality $C^{n-*} \simeq C$. In more modern language, Mishchenko had in effect introduced the symmetric L-groups $L^{n}(A)$, as the cobordism groups of $n$-dimensional symmetric Poincaré complexes over $A$. The symmetric signature of an $n$ dimensional Poincaré duality space $M$ with $\pi_{1}(M)=\Gamma$ is the cobordism 
class $\sigma^{*}(M) \in L^{n}(\mathbb{Z}[\Gamma])$ of the chain complex $C(\widetilde{M})$ of the universal cover $\widetilde{M}$. This is a homotopy invariant of $M$, which for $n \equiv 0(\bmod 4), \Gamma=\{1\}$, is just the ordinary signature. The symmetrization maps $1+T: L_{*}(A) \rightarrow$ $L^{*}(A)$ from the Wall quadratic $L$-groups $L_{*}(A)$ are isomorphisms modulo 2 -primary torsion, for any ring with involution $A$. The symmetrization of the surgery obstruction $\sigma_{*}(f, b) \in L_{n}(\mathbb{Z}[\Gamma])$ of an $n$-dimensional normal map $(f, b): N \rightarrow M$ is the difference of the symmetric signatures

$$
(1+T) \sigma_{*}(f, b)=\sigma^{*}(N)-\sigma^{*}(M) \in L^{n}(\mathbb{Z}[\Gamma])
$$

Mishchenko and Soloviev ([Mis5], [MisS1]) used sheaves of symmetric Poincaré complexes to define assembly maps ${ }^{12}$

$$
A: H_{*}(M ; \mathbb{L} \bullet(\mathbb{Z})) \rightarrow L^{*}(\mathbb{Z}[\Gamma])
$$

Here, $\mathbb{L} \bullet(\mathbb{Z})$ is the spectrum of the symmetric $L$-theory of $\mathbb{Z}$, and

$$
H_{*}(M ; \mathbb{L} \bullet(\mathbb{Z})) \otimes \mathbb{Q} \cong \sum_{k=0}^{\infty} H_{*-4 k}(M ; \mathbb{Q})
$$

The surgery obstruction of a normal map $(f, b): N \rightarrow M$ of closed $n$ dimensional manifolds is determined modulo 2-primary torsion by an element $[f, b]^{\bullet} \in H_{n}\left(M ; \mathbb{L}^{\bullet}(\mathbb{Z})\right)^{13}$. If $u: M \rightarrow B \Gamma$ is the classifying map for the universal cover of $M$, the assembly map $A$ for $M$ factors as

$$
A: H_{n}(M ; \mathbb{L} \bullet(\mathbb{Z})) \stackrel{u_{*}}{\longrightarrow} H_{n}(B \Gamma ; \mathbb{L} \bullet(\mathbb{Z})) \stackrel{A_{\Gamma}}{\longrightarrow} L^{n}(\mathbb{Z}[\Gamma])
$$

with $A_{\Gamma}$ the assembly map for the classifying space $B \Gamma$, and

$$
A[f, b]^{\bullet}=A_{\Gamma} u_{*}[f, b]^{\bullet}=(1+T) \sigma_{*}(f, b)=\sigma^{*}(N)-\sigma^{*}(M) \in L^{n}(\mathbb{Z}[\Gamma])
$$

is the difference of the symmetric signatures. The torsion-free part

$$
\begin{aligned}
u_{*}[f, b] \bullet 1 \in H_{n}(B \Gamma ; \mathbb{L} \bullet & \mathbb{Z})) \otimes \mathbb{Q} \\
& =H_{n-4 *}(B \Gamma ; \mathbb{Q})=\operatorname{Hom}_{\mathbb{Q}}\left(H^{n-4 *}(B \Gamma ; \mathbb{Q}), \mathbb{Q}\right)
\end{aligned}
$$

\footnotetext{
${ }^{12}$ This construction of $A$ required $M$ to be a manifold, which is not necessary in the construction of $A$ due to Ranicki [Ran9]; cf. the section below on surgery spectra.

${ }^{13}$ The symmetric $L$-theory homology class $[f, b]^{\bullet}$ does not depend on the bundle map $b$, being the difference $[f, b]^{\bullet}=f_{*}[N]_{\mathbb{L}}-[M]_{\mathbb{L}}$ of absolute invariants with $A[M]_{\mathbb{L}}=\sigma^{*}(M)$, $A[N]_{\mathbb{L}}=\sigma^{*}(N)$. See Ranicki $([\operatorname{Ran} 9],[\operatorname{Ran} 10])$ for the symmetric $L$-theory orientation of manifolds. The actual surgery obstruction is the quadratic $L$-theory assembly $\sigma_{*}(f, b)=$ $A[f, b] \bullet \in L_{n}(\mathbb{Z}[\Gamma])$ of a quadratic $L$-theory homology class $[f, b] \bullet \in H_{n}\left(M ; \mathbb{L}_{\bullet}\right)$ with $(1+T)[f, b]_{\bullet}=[f, b]^{\bullet}$, which is not in general the difference of absolute invariants.
} 
determines and is determined by the differences of the higher signatures

$$
\operatorname{signature}_{x}(N, u \circ f)-\operatorname{signature}_{x}(M, u) \in \mathbb{Q}, \quad x \in H^{n-4 *}(B \Gamma ; \mathbb{Q}) .
$$

If $f: N \rightarrow M$ is a homotopy equivalence of closed manifolds, then $\sigma^{*}(N)=$ $\sigma^{*}(M)$ and

$$
u_{*}[f, b] \bullet \operatorname{ker}\left(A_{\Gamma}: H_{n}\left(B \Gamma ; \mathbb{L}^{\bullet}(\mathbb{Z})\right) \rightarrow L^{n}(\mathbb{Z}[\Gamma])\right)
$$

Thus if $A_{\Gamma}$ is a rational injection then the higher signatures of $M$ and $N$ are equal and the Novikov Conjecture on the homotopy invariance of the higher signatures holds for $\Gamma$. In fact, the following is true :

Proposition. The Novikov Conjecture holds for a group $\Gamma$ if and only if the assembly map in symmetric L-theory

$$
A_{\Gamma}: H_{*}(B \Gamma ; \mathbb{L} \bullet(\mathbb{Z})) \rightarrow L^{*}(\mathbb{Z}[\Gamma])
$$

is a rational split injection.

Many proofs of the Novikov Conjecture use special properties of some class of groups $\Gamma$ to construct (rational) splittings $L^{*}(\mathbb{Z}[\Gamma]) \rightarrow H_{*}(B \Gamma ; \mathbb{L} \bullet(\mathbb{Z}))$ of $A_{\Gamma}$.

\section{Lusztig and the analytic approach}

In his thesis, published in 1972 [Lus], Lusztig made a major contribution to the theory of the Novikov Conjecture by being the first one to use analysis, more specifically, index theory, to attack the conjecture. Lusztig's paper was in fact the prototype for what was ultimately to be the largest body of literature related to the conjecture. The basic idea of Lusztig's work was to relate the higher signatures of a manifold to a priori homotopy invariants coming from the de Rham complex with local coefficients in a flat vector bundle. In the case of an oriented closed manifold $M^{2 m}$ with free abelian fundamental group $\mathbb{Z}^{k}$, the flat line bundles over $M$ are parametrized by a torus $T^{k}$, and a choice of a Riemannian metric on $M$ gives rise to a signature operator $D=d+d^{*}$ which can be "twisted" by any of these line bundles. Twisting by a line bundle does not change the index of $D$, which is just signature $(M)$, but viewing all the twists simultaneously gives a family of elliptic operators on $M$ parametrized by $T^{k}$. Lusztig showed that the index of this family, in the sense of the Atiyah-Singer Index Theorem for Families, is on the one hand a homotopy invariant, but on the other hand related to the higher signatures. He was thus able to give an analytic proof of 
the Novikov Conjecture for manifolds with free abelian fundamental group. His methods also gave partial results for other fundamental groups with "lots" of finite-dimensional representations (for which one can again twist the signature operator by a family of flat bundles).

\section{Splitting theorems for polynomial extensions}

Surgery on codimension 1 submanifolds has been an important feature of the study of non-simply-connected manifolds in general, and the Novikov Conjecture in particular. Browder ([Br2], [Br3]) used surgery on codimension 1 submanifolds to deal with the homotopy properties of simply-connected open manifolds, and non-simply-connected closed manifolds with $\pi_{1}=\mathbb{Z}$. Novikov used an iteration of codimension 1 surgeries to prove the topological invariance of the rational Pontrjagin classes. On the algebraic side, codimension 1 surgery corresponds to the algebraic $K$ - and $L$-theory properties of polynomial rings and their generalizations, starting with $\mathbb{Z}[\mathbb{Z}]=\mathbb{Z}\left[z, z^{-1}\right]$. We have already seen (in footnote 7 above) that Novikov in his Izvestia paper recognized the significance for his conjecture of the "fundamental theorem of algebraic $K$-theory" proved by Bass, Heller and Swan [BHS] and Bass [Bass]: for any ring $A$

$$
K_{1}\left(A\left[z, z^{-1}\right]\right) \cong K_{1}(A) \oplus K_{0}(A) \oplus \widetilde{\operatorname{Nil}_{0}}(A) \oplus \widetilde{\operatorname{Nil}_{0}}(A)
$$

with $\widetilde{\mathrm{Nil}}_{0}(A)$ the nilpotent class group. Farrell-Hsiang ([FarHs1], [FarHs3]) gave a geometric interpretation of the fundamental theorem in terms of splitting homotopy equivalences of manifolds $N \rightarrow M \times S^{1}$ along the codimension 1 submanifold $M \times\{*\} \subset M \times S^{1}$, with

$$
A=\mathbb{Z}\left[\pi_{1}(M)\right], A\left[z, z^{-1}\right]=\mathbb{Z}\left[\pi_{1}\left(M \times S^{1}\right)\right]=\mathbb{Z}\left[\pi_{1}(M) \times \mathbb{Z}\right] .
$$

Shaneson ([Sh1], [Sh2]) used this codimension 1 splitting theorem to give a geometric proof of the analogous $L$-theory splitting theorem

$$
L_{n}^{s}(\mathbb{Z}[\Gamma \times \mathbb{Z}]) \cong L_{n}^{s}(\mathbb{Z}[\Gamma]) \oplus L_{n-1}^{h}(\mathbb{Z}[\Gamma])
$$

for any finitely presented group $\Gamma$. Novikov [Nov10] gave an algebraic proof of the $L$-theory splitting theorem modulo 2-torsion:

$$
L_{n}\left(A\left[z, z^{-1}\right]\right) \otimes \mathbb{Z}[1 / 2] \cong\left(L_{n}(A) \oplus L_{n-1}(A)\right) \otimes \mathbb{Z}[1 / 2]
$$

for any ring with involution $A$ with $1 / 2 \in A$, with the involution extended by $\bar{z}=z^{-1}$. This splitting was used to give the algebraic proof of the Novikov Conjecture for free abelian groups in [Nov10]. Farrell and Hsiang 
[FarHs3] gave the corresponding geometric proof. The 2-torsion restrictions were removed by Ranicki ([Ran1], [Ran2]), and the splitting theorems

$$
\begin{aligned}
& L_{n}^{s}\left(A\left[z, z^{-1}\right]\right) \cong L_{n}^{s}(A) \oplus L_{n-1}^{h}(A) \\
& L_{n}^{h}\left(A\left[z, z^{-1}\right]\right) \cong L_{n}^{h}(A) \oplus L_{n-1}^{p}(A)
\end{aligned}
$$

were obtained algebraically for any ring with involution $A$, with $L_{*}^{s}(A)$ (resp. $\left.L_{*}^{h}(A), L_{*}^{p}(A)\right)$ the simple (resp. free, projective) $L$-groups. The simple $L$ groups $L_{*}^{s}(A)$ are the original surgery obstruction groups of Wall [Wall2]; there is only a 2-primary torsion difference between $L_{*}^{s}(A), L_{*}^{h}(A)$ and $L_{*}^{p}(A)$. The lower $K$-groups $K_{-i}(A)$ and the lower $N K$-groups $N K_{-i}(A)$ were defined by Bass [Bass] for any ring $A$, to fit into splittings

$$
K_{-i+1}\left(A\left[z, z^{-1}\right]\right) \cong K_{-i+1}(A) \oplus K_{-i}(A) \oplus N K_{-i+1}(A) \oplus N K_{-i+1}(A)
$$

for all $i \geq 0$, with $N K_{1}(A)=\widetilde{\operatorname{Nil}}_{0}(A)$. The analogous lower $L$-groups $L^{\langle-i\rangle}(A)$ were defined in [Ran2] for any ring with involution, to fit into splittings

$$
L_{n}^{\langle-i+1\rangle}\left(A\left[z, z^{-1}\right]\right) \cong L_{n}^{\langle-i+1\rangle}(A) \oplus L_{n-1}^{\langle-i\rangle}(A)
$$

for all $i \geq 0$, with $L_{*}^{\langle 0\rangle}(A)=L_{*}^{p}(A)$. The forgetful maps $L_{n}^{\langle-i+1\rangle}(A) \rightarrow$ $L_{n}^{\langle-i\rangle}(A)$ are isomorphisms modulo 2-primary torsion, with the relative terms the Tate $\mathbb{Z}_{2}$-cohomology of the duality involution on $K_{-i}(A)$.

\section{Cappell and codimension 1 splitting theorems}

With the development of surgery theory for non-simply connected manifolds, machinery was finally in place that could be used to determine when a homotopy equivalence $f: M^{\prime} \rightarrow M$ of closed manifolds "splits" with respect to submanifold $P$ of $M$, in other words, when it can be deformed so as to restrict to a homotopy equivalence $P^{\prime} \rightarrow P$. The general splitting obstruction theory was worked out by Wall [Wall2, §12]. As noted in the previous section, the case where $P$ is of codimension 1 in $M$ (i.e. a hypersurface) is of particular importance for Novikov Conjecture. Suppose $P$ is a separating hypersurface in $M$, so that $M$ is the union of two codimensionzero compact submanifolds, $M_{+}$and $M_{-}$, each with boundary $P$. Then assuming that $f$ splits, we get a comparable decomposition of $M^{\prime}$ as the union of two submanifolds $M_{+}^{\prime}$ and $M_{-}^{\prime}$, each with boundary $P^{\prime}$. By Van Kampen's Theorem, $\pi_{1}(M)$ splits as an amalgamated free product:

$$
\pi_{1}(M)=\pi_{1}\left(M_{+}\right) *_{\pi_{1}(P)} \pi_{1}\left(M_{-}\right)
$$


So the question of whether or not $f$ preserves higher signatures can be reduced to questions about the restrictions of $f$ to the various pieces of $M^{\prime}$, and about higher signatures for the groups $\pi_{1}\left(M_{+}\right), \pi_{1}(P), \pi_{1}\left(M_{-}\right)$. If these groups are simpler than $\pi_{1}(M)$, there is some hope to use this strategy to give an inductive proof of the Novikov Conjecture for a large class of groups that can be built up from amalgamated free products. As we saw above, Novikov was certainly aware that such a strategy might be useful, especially for studying polycyclic groups, but Cappell ([Cap2], [Cap3]) was the one to finally work out the applications to the Novikov Conjecture using both this case of amalgamated free products (corresponding geometrically to separating hypersurfaces) and the case of $H N N$ extensions (corresponding geometrically to non-separating hypersurfaces, generalizing the polynomial extensions considered in the previous section). Cappell's theory was the first successful attempt to inductively verify the Novikov Conjecture for a large class of well-behaved fundamental groups. An unexpected subtlety which Cappell had to overcome was the "UNil obstruction" to splitting, involving the $L$-theoretic analogues of the nilpotent class group $\widehat{\mathrm{Nil}_{0}}$; since this involves only 2-torsion it has little impact on the higher signatures, but it does play an important role in any attempts to correctly formulate an integral Novikov Conjecture.

\section{Mishchenko and Fredholm representations}

Meanwhile, in the wake of Lusztig's thesis, others hoped to use index theory to attack the Novikov Conjecture for large numbers of fundamental groups. But Lusztig's methods required having families of flat vector bundles, which may not be available for non-commutative groups. Mishchenko ([Mis3], [Mis4]) suggested an important idea for overcoming this difficulty, namely the use of Fredholm representations of the fundamental group. A Fredholm representation $\rho$ of a group $\Gamma$ on a $(\mathbb{Z} / 2$-graded) Hilbert space $\mathcal{H}$ is a pair $\left(\rho^{0}, \rho^{1}\right)$ of unitary representations of $\Gamma$ on $\mathcal{H}^{(0)}$ and on $\mathcal{H}^{(1)}$, respectively, together with a Fredholm operator $T: \mathcal{H}^{(0)} \rightarrow \mathcal{H}^{(1)}$ which intertwines the two representations modulo compact operators. One should think of $\rho$ as being the formal difference $\rho^{0}-\rho^{1}$, which one can think of as being approximately finite-dimensional, even though $\rho^{0}$ and $\rho^{1}$ are themselves infinite-dimensional, so that the case where $T$ is a precise intertwiner is uninteresting. A general discrete group $\pi$ always has lots of Fredholm representations, even though it may have very few finite-dimensional representations. We can think of these as parameterizing certain generalized flat vector bundles over manifolds with $\Gamma$ as fundamental group. Mishchenko's idea was to prove the appropriate index theorem for the signature operator with coefficients in a Fredholm representation of the fundamental group, 
then to substitute these for genuine flat vector bundles in Lusztig's machinery.

Another of Mishchenko's major contributions was to notice that this program works especially well in the presence of a "non-positive curvature assumption" in a model for $B \Gamma$, when for example $B \Gamma$ is a compact manifold whose universal cover $E \Gamma$ is a locally symmetric space of non-compact type. Then the "outward-pointing vector field" on $E \Gamma$ "asymptotically commutes" with covering translations by $\Gamma$, and thus gives rise to what Connes later called the "dual Dirac" operator. By using the machinery of Fredholm representations and the dual Dirac (or its analogue in the case of Bruhat-Tits buildings), Mishchenko and his co-workers ([Mis3], [Mis4], [Mis5], [Mis6], [MisS2], [MisS3]) were able to verify the Novikov Conjecture for a number of geometrically interesting fundamental groups.

\section{Farrell-Hsiang and the geometric topology approach}

At about the same time, Farrell and Hsiang embarked on a program to systematically attack not only the Novikov Conjecture but also the Borel Conjecture for classes of groups of geometric interest, using methods of geometric topology. Farrell and Hsiang began [FarHs3] by proving both conjectures for free abelian fundamental groups by purely topological methods, using splitting machinery growing out of Farrell's thesis work [Far] on when a manifold fibers over a circle. Then they went on to study the conjectures for flat manifolds (Bieberbach groups) ([FarHs4], [FarHs6], [FarHs7]), nonpositively curved manifolds ([FarHs8], [FarHs10]), and almost flat manifolds (infra-nilpotent groups) [FarHs11]. Of special interest in their work was a new idea which they applied to the study of the Novikov Conjecture for Bieberbach groups [FarHs7]: the application of "controlled" topology. The rough idea of how Farrell and Hsiang applied this, reformulated in terms of a fundamental theorem of Chapman and Ferry $[\mathrm{ChapF}],{ }^{14}$ is the following. The Chapman-Ferry " $\alpha$-approximation theorem" says that given a closed manifold $M^{n}$, with $n>4$, there is a constant $\varepsilon>0$ such that a homotopy equivalence $f: M^{\prime} \rightarrow M$ of closed manifolds is homotopic to a homeomorphism provided that there is a homotopy inverse $g: M \rightarrow M^{\prime}$ to $f$ such that the homotopies from $f g$ and $g f$ to the identity maps don't move points more than a distance $\varepsilon$ (as measured in $M$ ). Suppose one has a homotopy equivalence $f: M^{\prime} \rightarrow M$, and suppose the fundamental group of $M$ is such that there exist coverings $M \rightarrow M$ of arbitrarily large degree, which stretch distances by an arbitrarily large amount. Then lifting $f$ by such coverings, one can get new homotopy equivalences $M^{\prime} \rightarrow M$ which are "controlled"

\footnotetext{
${ }^{14}$ Farrell and Hsiang actually quoted earlier papers of Chapman and of Ferry that use some of the same ideas.
} 
as much as one likes, so that eventually these are homotopic to homeomorphisms. This is a major step in proving the Borel Conjecture. For further discussion of this and other geometric approaches to rigidity, see the subsections on "Farrell-Hsiang, Ferry-Weinberger and tangentiality" and on "The Farrell-Jones Program."

\section{Kasparov and operator-theoretic $K$-homology}

Still another analytic attack on the Novikov Conjecture, motivated both by Lusztig's thesis and by ideas of Atiyah and Singer concerning possible reformulations of index theory, was begun by Gennadi Kasparov in the 1970's. The idea behind this program was to give a good analytic model for the homology theory dual to $K$-theory, so that elliptic operators on manifolds would naturally give rise to $K$-homology classes. Then the index of an elliptic operator is computed merely by taking the image, in $K$-homology of a point, of the corresponding $K$-homology class. By analyzing the $K$ homology class of the signature operator on a non-simply connected manifold, one could hope to redo what Lusztig had done, but in a more powerful setting. Kasparov's earliest results in this direction, as well as the first announcements of his results on the Novikov Conjecture, appeared in [Kas1] and [Kas2], although the power of his methods did not become clear until the development of the " $K K$ calculus" in [Kas3]. (For more informal expositions, see also [Black] and [Fack1].) While Mishchenko's Fredholm representations were basically equivalent to $K$-homology classes in the Kasparov sense, Kasparov's "intersection product" in $K K$ gave more powerful technical tools for overcoming a drawback of Mishchenko's method pointed out in [HsR]. In his famous "Conspectus" [Kas4], Kasparov for the first time was able to sketch a complete analytic proof of a result not yet provable by purely topological methods: that the Novikov Conjecture (in fact, even an integral version, after localizing away from the prime 2) holds for groups $\Gamma$ which are fundamental groups of complete Riemannian manifolds of non-positive curvature, or which can be realized as discrete subgroups of connected Lie groups.

\section{Surgery spectra and assembly (Quinn)}

The global approach to surgery theory initiated by Sullivan and Wall was carried forward by Quinn, and has proved useful in attacking the Borel and Novikov Conjectures using various mixtures of geometry and algebra. Given an $n$-dimensional Poincaré duality space $M$, let $\mathcal{S}^{T O P}(M)$ be the topological manifold structure set of $M$, consisting of the equivalence classes of pairs $(N, f)$ with $N$ a closed $n$-dimensional topological manifold and $f: N \rightarrow M$ a homotopy equivalence. Two such pairs $(N, f),\left(N^{\prime}, f^{\prime}\right)$ are equivalent 
if there exists an $h$-cobordism $\left(W ; N, N^{\prime}\right)$ with a homotopy equivalence $\left(g ; f, f^{\prime}\right):\left(W ; N, N^{\prime}\right) \rightarrow M \times(I ;\{0\},\{1\})$. The Browder-Novikov-SullivanWall theory provides an obstruction theory for deciding if $\mathcal{S}^{T O P}(M)$ is non-empty, and for manifold $M$ there is an exact sequence of pointed sets

$$
\cdots \rightarrow L_{n+1}(\mathbb{Z}[\Gamma]) \rightarrow \mathcal{S}^{T O P}(M) \stackrel{\theta}{\rightarrow}[M, G / T O P] \stackrel{A}{\rightarrow} L_{n}(\mathbb{Z}[\Gamma])
$$

with $\Gamma=\pi_{1}(M)$, and $G / T O P$ the homotopy fiber of the forgetful map $J$ from the classifying space BTOP for stable topological bundles to the classifying space $B G$ for stable spherical fibrations. For a manifold $M$ the map $\theta: \mathcal{S}^{T O P}(M) \rightarrow[M, G / T O P]$ sends an element $(N, f)$ of the structure set to the difference between the stable normal bundle of $M$ and the push-forward under $f$ of the stable normal bundle of $N$. The map $A:[M, G / T O P] \rightarrow L_{n}(\mathbb{Z}[\Gamma])$ sends an element of $[M, G / T O P]$, represented by a topological bundle $\eta$ over $M$ with a fiber homotopy trivialization, to the surgery obstruction $\sigma_{*}(f, b) \in L_{n}(\mathbb{Z}[\Gamma])$ of any normal map $(f, b): N \rightarrow M$ with $b: \nu_{N} \rightarrow \eta$. The interpretation of $G / T O P$ as a surgery classifying space came from the work of Casson and Sullivan on the manifold Hauptvermutung, which grew out of Novikov's proof of the topological invariance of the rational Pontrjagin classes ([Ran10], [Ran11]).

Quinn ([Q1], [Q2], [Q3]) constructed for each space $X$ a spectrum $\mathbb{L}_{\bullet}(X)$ consisting of normal maps with a reference map to $X$, such that the homotopy groups are the surgery obstruction groups

$$
\pi_{*}\left(\mathbb{L}_{\bullet}(X)\right)=L_{*}\left(\mathbb{Z}\left[\pi_{1}(X)\right]\right)
$$

and with a homotopy equivalence

$$
\mathbb{L}_{\bullet}(\text { pt. })_{0} \simeq L_{0}(\mathbb{Z}) \times G / T O P .
$$

The surgery obstruction map $A$ for an $n$-dimensional topological manifold $X$ was interpreted as the abelian group morphism

$$
A:[X, G / T O P] \subseteq\left[X, L_{0}(\mathbb{Z}) \times G / T O P\right]=H_{n}\left(X ; \mathbb{L}_{\bullet}(\mathrm{pt} .)\right) \stackrel{A}{\longrightarrow} L_{*}\left(\mathbb{Z}\left[\pi_{1}(X)\right]\right)
$$

defined by the geometric surgery assembly map. Let $\mathbb{H} \bullet\left(X ; \mathbb{L}_{\bullet}(p t).\right)$ be the spectrum with homotopy groups the generalized homology groups with $\mathbb{L}_{\bullet}$ (pt.)-coefficients

$$
\pi_{*}\left(\mathbb{H}_{\bullet}\left(X ; \mathbb{L}_{\bullet}(\mathrm{pt} .)\right)\right)=H_{*}\left(X ; \mathbb{L}_{\bullet}(\mathrm{pt} .)\right)
$$

The surgery assembly map is induced by a map of $\operatorname{spectra} A: \mathbb{H} \bullet\left(X ; \mathbb{L}_{\bullet}(\right.$ pt. $\left.)\right)$ $\rightarrow \mathbb{L}_{\bullet}(X)$. For the surgery exact sequence it is necessary to work with the 
1-connective simply-connected surgery spectrum $\mathbb{L}_{\bullet}=\mathbb{L}_{\bullet}\langle 1\rangle$ (pt.) such that $\left(\mathbb{L}_{\bullet}\right)_{0} \simeq G / T O P$. The cofibre $\mathbb{S}_{\bullet}(X)$ of the spectrum-level 1-connective surgery assembly map fits into a (co)fibration sequence

$$
\mathbb{H}_{\bullet}\left(X ; \mathbb{L}_{\bullet}\right) \stackrel{A}{\rightarrow} \mathbb{L}_{\bullet}(X) \rightarrow \mathbb{S}_{\bullet}(X)
$$

and the homotopy groups $\pi_{*}\left(\mathbb{S}_{\bullet}(X)\right)=\mathbb{S}_{*}(X)$ fit into a long exact sequence of abelian groups

$$
\cdots \rightarrow \mathbb{S}_{n+1}(X) \rightarrow H_{n}\left(X ; \mathbb{L}_{\bullet}\right) \stackrel{A}{\rightarrow} L_{n}\left(\mathbb{Z}\left[\pi_{1}(X)\right]\right) \rightarrow \mathbb{S}_{n}(X) \rightarrow \ldots
$$

There is such a sequence both for the free $L$-groups $L_{*}=L_{*}^{h}$ and for the simple $L$-groups $L_{*}=L_{*}^{s}$. If $M$ is a closed $n$-dimensional TOP manifold then

$$
[M, G / T O P] \cong H^{0}\left(M ; \mathbb{L}_{\bullet}\right) \cong H_{n}\left(M ; \mathbb{L}_{\bullet}\right)
$$

and the structure set

$$
\mathcal{S}^{T O P}(M)=\mathbb{S}_{n+1}(M)
$$

have abelian group structures, as indeed do all the rel $\partial$ structure sets

$$
\mathcal{S}^{T O P}\left(M \times D^{k} \operatorname{rel} \partial\right)=\mathbb{S}_{n+k+1}(M)(k \geq 0) .
$$

Nicas [Ni1] used the abelian group structures to prove induction theorems for the structure set. If $\left(M^{n}, \partial M\right)$ is an $n$-dimensional manifold with boundary and $\pi_{1}(M)=\Gamma$, then Siebenmann Periodicity [KirS $]^{15}$ shows that there is a monomorphism $\mathcal{S}^{T O P}\left(M^{n}\right) \rightarrow \mathcal{S}^{T O P}\left(M^{n} \times D^{4}\right.$ rel $\left.\partial\right), n \geq 6$, which is an isomorphism for $\partial M \neq \emptyset$.

\section{Work related to the Novikov Conjecture: The Last 12 Years or So, I: Homotopy Theory and Algebra}

\section{Algebraic surgery theory (Ranicki)}

It was already suggested by Wall [Wall2] that a development of chain complexes with Poincaré duality would be the appropriate formulation for the 'whole setup' of surgery. The symmetric Poincaré complex theory of Mishchenko [Mis2] was extended by Ranicki ([Ran4], [Ran5], [Ran 7]) to a comprehensive theory of chain complexes with Poincaré duality, including the quadratic structures required for the Wall surgery obstruction. The surgery obstruction of an $n$-dimensional normal map $(f, b): M \rightarrow X$ was

\footnotetext{
${ }^{15}$ See $[\mathrm{Ni1}]$ and $[\mathrm{CaW} 1]$ for a correction.
} 
expressed as a quadratic Poincaré cobordism class $\sigma_{*}(f, b) \in L_{n}\left(\mathbb{Z}\left[\pi_{1}(X)\right]\right)$ of the quadratic Poincaré duality on the algebraic mapping cone $C\left(f^{!}\right)$of the Umkehr $\mathbb{Z}\left[\pi_{1}(X)\right]$-module chain map

$$
f^{!}: C(\widetilde{X}) \simeq C(\widetilde{X})^{n-*} \stackrel{f^{*}}{\longrightarrow} C(\widetilde{M})^{n-*} \simeq C(\widetilde{M}) .
$$

The main application of the theory to the Novikov Conjecture is by way of the algebraic surgery assembly map, as follows.

Ranicki ([Ran6], [Ran7], [Ran8], [Ran9], [LeRa]) used quadratic Poincaré complexes to define, for any ring with involution $R$, an algebraic surgery spectrum $\mathbb{L}_{\bullet}(R)$ such that $\pi_{*}\left(\mathbb{L}_{\bullet}(R)\right)=L_{*}(R)$, and an algebraic surgery assembly map

$$
A: H_{*}\left(X ; \mathbb{L}_{\bullet}(\mathbb{Z})\right) \rightarrow L_{*}\left(\mathbb{Z}\left[\pi_{1}(X)\right]\right)
$$

for any space $X$. These are algebraic versions of Quinn's geometric constructions, particularly the surgery exact sequence. Taylor and Williams [TW] determined the homotopy types of the algebraic $L$-spectra, generalizing Sullivan's determination of the homotopy type of $G / T O P$.

Proposition. The Novikov Conjecture holds for a group $\Gamma$ if and only if the algebraic surgery assembly map $A_{\Gamma}: H_{*}\left(B \Gamma ; \mathbb{L}_{\bullet}(\mathbb{Z})\right) \rightarrow L_{*}(\mathbb{Z}[\Gamma])$ is a rational split injection.

Proposition. The h-cobordism Borel Conjecture holds for a group $\Gamma$ if and only if the algebraic surgery assembly map $A_{\Gamma}: H_{*}\left(B \Gamma ; \mathbb{L}_{\bullet}(\mathbb{Z})\right) \rightarrow L_{*}^{h}(\mathbb{Z}[\Gamma])$ is an isomorphism. Similarly for the $s$-cobordism Borel Conjecture with $L_{*}^{s}$.

See [Ran10] for a somewhat more detailed account. The algebraic surgery assembly map is a special case of the universal assembly construction of Weiss and Williams ([WW3], [WW4]). Study of the assembly map for polynomial extensions and amalgamated free products ([MilgR], [Ran8], [Ran10]) has been used to prove some special cases of the (integral) Novikov Conjecture, extending the method of Cappell.

\section{The homotopy-limit problem, descent (Carlsson)}

The homotopy theoretic approach to the Novikov Conjecture is based on experience with analogous problems arising with finite groups. We first recall G. B. Segal's equivariant stable theory. For any finite group $G$, Segal constructs a $G$-space $Q^{G}\left(S^{0}\right)$, whose fixed point set is described as

$$
\prod_{K \subseteq G} Q\left(B W_{G}(K)_{+}\right)
$$


where $Q$ denotes $\Omega^{\infty} \Sigma^{\infty}, W_{G}(K)$ denotes the "Weyl group" $N_{G}(K) / K$, and "+" denotes disjoint basepoint added. The factor corresponding to the trivial subgroup is thus $Q\left(B G_{+}\right)$. This factor can be seen to be the image of a certain transfer map, which is formally similar to the assembly in its definition. The affirmative solution of Segal's Burnside ring conjecture now shows that in the case of a finite $p$-group, this factor includes as a factor in the homotopy fixed set of the $G$-action on $Q^{G}\left(S^{0}\right)$ after $p$-adic completion. See [Car1] or [Car2] for a more thorough discussion. Similarly, it follows from results of Atiyah [At1] that the assembly map for $K$-theory of the complex group ring of a finite $p$-group is split injective, indeed that it is an equivalence, after $p$-adic completion. Here it is crucial that we consider the periodic complex $K$-theory. In this case, the inverse to the assembly map is given by the natural map to the homotopy fixed set of the action of $G$ on $B U$.

Both these constructions suggest that if one wants to study the assembly map more generally, one should attempt to construct a splitting map using the homotopy fixed set of an action of the group $\Gamma$ in question on the $K$ theory of the coefficient ring. In the finite group cases mentioned above, the existence of the corresponding $G$-action and homotopy fixed set was self-evident, arising from the actions of the $G$ by conjugation on symmetric groups or complex matrix groups. In the case of infinite groups of geometric interest, the obvious actions on rings of infinite matrices yields nothing, since the $K$-theory of infinite matrix rings is trivial by an appropriate use of the "Eilenberg swindle". It turns out, though, that the Pedersen-Weibel bounded $K$-theory $[\mathrm{PeW}]$ gives the right model. For any torsion-free group $\Gamma$, it is possible to construct a bounded $K$-theory spectrum $K^{\Gamma}$ on which $\Gamma$ acts with fixed point set equal to the algebraic $K$-theory of the group ring. Furthermore, it is possible to construct an equivariant assembly map $\alpha$ from the "locally finite homology" of the universal cover of $B \Gamma$ to $K^{\Gamma}$, whose induced map on fixed-point sets is the usual assembly. When one can prove that the map $\alpha$ is an equivalence of spectra, standard facts about homotopy fixed-point sets allow one to conclude that the usual assembly map is onto a wedge factor. See [Car3] for an expository discussion of this. Details are done in [Car5]. There, the bounded $K$-theory is computed for $G / K$, where $G$ is a connected Lie group and $K$ is its maximal compact subgroup. This allows one to prove the integral form of the Novikov Conjecture for cocompact torsion-free discrete subgroups of Lie groups. Similar methods work for the case of cocompact torsion-free discrete subgroups of $p$-adic Lie groups, as in the Princeton thesis of P. Mostad. 


\section{The Carlsson-Pedersen approach}

An alternative is to observe that one perhaps does not need to compute the bounded $K$-theory explicitly, but only to produce a $\Gamma$-spectrum to which the bounded $K$-theory maps equivariantly, and so that the composite of this map with the map $\alpha$ above is an equivalence. This is the approach taken in $[\mathrm{CarP}]$, where it is assumed that the universal cover of the classifying space can be compactified to a contractible space with $\Gamma$-action, with certain hypotheses on the action on the boundary. The method of Carlsson and Pedersen combines this equivariant homotopy theory with the categorical approach of Pedersen and Weibel $[\mathrm{PeW}]$, the continuously controlled categories of Anderson, Connolly, Ferry and Pedersen [AnCFP], and the lower $L$-theory of Ranicki [Ran10]. (See the section below on bounded and controlled topology for more information.) In particular, Carlsson and Pedersen proved the integral form of the Novikov Conjecture for, e.g., Gromov's word-hyperbolic groups. They are currently in the process of extending this work to the case of groups which are not torsion-free, using analogues of the Baum-Connes ideas discussed below.

\section{Controlled, continuously controlled and bounded topology}

Controlled topology gives geometric methods for approximating homotopy equivalences by homeomorphisms $[\mathrm{ChapF}]$. As we shall see in the sections below on the work of Farrell-Jones and Ferry-Weinberger, these methods can be directly applied to proofs of the Novikov and Borel Conjectures. The controlled algebra of Quinn [Q3] uses a mixture of algebra and topology for recognizing certain types of spectra to be generalized homology spectra. In [Q4], Quinn gave applications to the algebraic $K$-theory of polycyclic groups. For certain groups $\Gamma$ it is possible to show that there is enough codimension 1 transversality to prove that the surgery spectrum $\mathbb{L}(\mathbb{Z}[\Gamma])$ is a generalized homology spectrum, verifying the Conjectures by showing that the assembly map $A_{\Gamma}: H_{*}\left(B \Gamma ; \mathbb{L}_{\bullet}(\mathbb{Z})\right) \rightarrow L_{*}(\mathbb{Z}[\Gamma])$ is an isomorphism. Yamasaki ([Ya1], [Ya2]) applied these methods to the case when $\Gamma$ is a crystallographic group. Other results, both positive and negative, on topological rigidity statements for crystallographic groups may be found in the work of Connolly and Koźniewski ([CyK1]-[CyK3]). Bounded topology also gives methods for recognizing generalized homology spectra, using the categorical methods initiated by Pedersen and Weibel [PW]. The most effective results on the Novikov Conjecture obtained algebraically use the continuously controlled category of Anderson, Connolly, Ferry and Pedersen $[\mathrm{AnCFK}]$ - see the section above on the work of Carlsson and Pedersen. For more details on how bounded and continuously controlled topology are related to the Novikov Conjecture, see the papers of Pedersen [Pe2] and 
Ferry-Weinberger [FW2].

\section{$K$-theoretic analogues of the Novikov and Borel Conjectures}

Proposition. If the Borel Conjecture holds for $\Gamma$ then the Whitehead group of $\Gamma$ vanishes, $\mathrm{Wh}(\Gamma)=0$.

Proof. Let $K$ be a finite aspherical polyhedron with $\pi_{1}(K)=\Gamma$. Let $M$ be a regular neighborhood of $K$ in some high-dimensional euclidean space. If $\tau \in \mathrm{Wh}(\Gamma)$, then we can build an $h$-cobordism rel boundary, $\left(W, M, M^{\prime}\right)$ so that $\tau(W, M)=\tau$. Let $f:\left(W, M, M^{\prime}\right) \rightarrow(M \times[0,1], M \times\{0\}, M \times\{1\})$ be a homotopy equivalence with $f \mid M \times\{0\}=i d$ and $f \mid W-M \times\{0,1\}$ a homeomorphism. Since $f \mid M^{\prime}$ is a homotopy equivalence, the Borel Conjecture implies that $f \mid M^{\prime}$ is homotopic to a homeomorphism rel boundary. By the homotopy extension theorem, we can assume that $f \mid M^{\prime}$ is a homeomorphism. Applying the conjecture again, $f$ is homotopic to a homeomorphism rel $\partial W$. Since Whitehead torsion is a topological invariant and $\tau(M \times[0,1], M \times\{0\})=0, \tau=0$.

Proposition. The Borel Conjecture holds for a group $\Gamma$ if and only if the algebraic surgery assembly map $A_{\Gamma}: H_{*}\left(B \Gamma ; \mathbb{L}_{\bullet}(\mathbb{Z})\right) \rightarrow L_{*}(\mathbb{Z}[\Gamma])$ is an isomorphism and $\mathrm{Wh}(\Gamma)=0$, with $L_{*}=L_{*}^{h}=L_{*}^{s}$.

Thus if the Borel Conjecture holds for $\Gamma$ then $\mathbb{S}_{*}(B \Gamma)=0$, and if $(M, \partial M)$ is an $n$-dimensional manifold with boundary such that $\pi_{1}(M)=\Gamma$ and $M$ is aspherical then

$$
\mathcal{S}^{T O P}(M \operatorname{rel} \partial)=\mathbb{S}_{n+1}(M)=\mathbb{S}_{n+1}(B \Gamma)=0
$$

Remark. The "fundamental theorem of $K$-theory" of Bass-Heller-Swan ([BHS], [Bass]) for the Whitehead group of a product $\Gamma \times \mathbb{Z}$ is

$$
\mathrm{Wh}(\Gamma \times \mathbb{Z})=\mathrm{Wh}(\Gamma) \oplus \widetilde{K}_{0}(\mathbb{Z}[\Gamma]) \oplus \widetilde{\mathrm{Nil}}_{0}(\mathbb{Z}[\Gamma]) \oplus \widetilde{\mathrm{Nil}_{0}}(\mathbb{Z}[\Gamma])
$$

Thus $\widetilde{K}_{0}(\mathbb{Z}[\Gamma])$ and $\widetilde{\operatorname{Nil}}_{0}(\mathbb{Z}[\Gamma])$ are direct summands in $\operatorname{Wh}(\Gamma \times \mathbb{Z})$, and these must vanish as well if the conjecture holds for $\Gamma \times \mathbb{Z}$. Similarly, the conjecture for products of $\Gamma$ with free abelian groups implies that all the negative $K$-groups of $\mathbb{Z}[\Gamma]$ must vanish.

Thus, rigidity for aspherical manifolds with boundary requires that the Whitehead groups and projective class groups of the fundamental groups of these manifolds should vanish. Consequently, a number of authors have proven vanishing theorems for Whitehead groups of fundamental groups of 
aspherical manifolds and polyhedra, beginning with the proof in $[\mathrm{Hi}]$ that $\mathrm{Wh}(\mathbb{Z})=0$ and in $[\mathrm{BHS}]$ that $\mathrm{Wh}\left(\mathbb{Z}^{n}\right)=0$. Other efforts along these lines include [FarHs2], [FarHs9], [FJ3], [FJ12], [Ni2], [Ni3], [Q4] and [Wald1]. A notable recent effort is the paper $[\mathrm{Hu}]$ in which $\mathrm{Hu}$ proves that $\mathrm{Wh}(\Gamma)=$ 0 when $\Gamma$ is the fundamental group of a finite nonpositively curved ( $\equiv$ CAT(0)) polyhedron. The paper uses a Gromov hyperbolization trick to reduce the problem to the case of a nonpositively curved $P L$ manifold. This case is then handled by an extension of the methods of [FJ13]. Hu also proves that the Whitehead group of $\Gamma$ vanishes for any $\Gamma$ which is isomorphic to a torsion-free cocompact discrete subgroup of $S L_{n}\left(\mathbb{Q}_{p}\right)$, where $\mathbb{Q}_{p}$ is the field of $p$-adic numbers.

There is an assembly map in algebraic $K$-theory, first introduced by Loday [Lod], which is analogous to the one for $L$-theory:

$$
A: H_{*}(B \Gamma ; \mathbb{K}(\mathbb{Z})) \rightarrow K_{*}(\mathbb{Z}[\Gamma])
$$

(For further information on how to understand this map, see [Wald1], §15.) In his address to the 1983 ICM, W.-C. Hsiang, [Hs2], proposed four conjectures as $K$-theory analogues of the Novikov and Borel Conjectures. The last of these refers to Loday's assembly map.

Conjecture 1. Let $\Gamma$ be a finitely presented group. Then $K_{-i}(\mathbb{Z}[\Gamma])=0$ for $i \geq 2$. At least, $K_{-i}(\mathbb{Z}[\Gamma])=0$ for $i \gg 0$.

Conjecture 2. Let $\Gamma$ be the fundamental group of a closed $K(\Gamma, 1)$-manifold. Then $\operatorname{Wh}(\Gamma)=\widetilde{K}_{0}(\mathbb{Z}[\Gamma])=K_{-i}(\mathbb{Z}[\Gamma])=0,(i \geq 1)$.

Conjecture 3. Let $\Gamma$ be a torsion-free group such that $B \Gamma$ has the homotopy type of a finite $C W$-complex. Then $\mathrm{Wh}(\Gamma)=\widetilde{K}_{0}(\mathbb{Z}[\Gamma])=K_{-i}(\mathbb{Z}[\Gamma])=$ $0,(i \geq 1)$.

Conjecture 4. If $\Gamma$ is a torsion-free group such that $B \Gamma$ is of the homotopy type of a finite complex, then

$$
A \otimes i d: H_{*}(B \Gamma ; \mathbb{K}(\mathbb{Z})) \otimes \mathbb{Q} \rightarrow K_{*}(\mathbb{Z}[\Gamma]) \otimes \mathbb{Q}
$$

is an isomorphism.

The first conjecture is somewhat tangential to the concerns of this survey and will not be discussed here, though it is true for finite groups by work of Carter [Carter]. The second is the $K$-theory part of the Borel Conjecture for closed aspherical manifolds. The third is a generalization of the second to 
the case of finite aspherical polyhedra. As we have seen, this must hold if the Borel Conjecture mentioned above is true for compact aspherical manifolds. The fourth conjecture, however, is a true analogue of the classical Novikov Conjecture, though it is only stated for a restricted class of groups. A still closer analogue of the classical Novikov Conjecture would be:

Conjecture 5. For any group $\Gamma$, the rational $K$-theory assembly map

$$
A \otimes i d: H_{*}(B \Gamma ; \mathbb{K}(\mathbb{Z})) \otimes \mathbb{Q} \rightarrow K_{*}(\mathbb{Z}[\Gamma]) \otimes \mathbb{Q}
$$

is injective.

The integral version of Conjecture 4 is:

Algebraic $K$-theory Isomorphism Conjecture. If $\Gamma$ is the fundamental group of a finite aspherical polyhedron, then the assembly map

$$
A: H_{i}(B \Gamma ; \mathbb{K}(\mathbb{Z})) \rightarrow K_{i}(\mathbb{Z}[\Gamma])
$$

is an isomorphism for all $i \geq 0$.

For $i=0$ this is just the conjecture that $\widetilde{K}_{0}(\mathbb{Z}[\Gamma])=0$, and for $i=1$ that $\mathrm{Wh}(\Gamma)=0$.

Results on the $K$-theory Isomorphism Conjecture for discrete subgroups of Lie groups and for groups satisfying non-negative curvature assumptions may be found in [FJ12] and [Car5]. For various technical reasons, it turns out that in approaching Conjecture 5, it is best to introduce Waldhausen's algebraic $K$-theory of spaces $A(X)$ [Wald2], a theory which has geometrical interpretations in terms of pseudo-isotopies of manifolds [Wald3]. There are analogues of the above conjectures for $A$-theory as well, results about which may be found in the papers by Carlsson, Carlsson-Pedersen, and FarrellJones already cited.

In a technical tour de force ([BöHM1], [BöHM2]), Bökstedt, Hsiang, and Madsen have proved Conjecture 5 for a class of groups including all groups $\Gamma$ such that $H_{i}(B \Gamma)$ is finitely generated in each dimension. This, of course, includes all groups of type $F P^{\infty}$ - groups $\Gamma$ such that $B \Gamma$ has finite $n$ skeleton for all $n$. Their argument is homotopy-theoretic and relies on both a topological version of cyclic homology theory and use of Waldhausen's A-theory. 


\section{Work related to the Novikov Conjecture: The Last 12 Years or So, II: Geometric Topology}

\section{Farrell-Hsiang, Ferry-Weinberger and tangentiality}

In [FarHs8], Farrell and Hsiang gave a proof of the integral Novikov Conjecture for closed Riemannian manifolds of nonpositive curvature. Their approach was to use Quinn's geometric description of the $L$-spectrum, [Q1], to construct an explicit splitting of the assembly map. An interesting feature of their construction is that it uses the nonpositive curvature assumption to produce a suitably nice compactification of the universal cover. The existence of this compactification is the only aspect of nonpositive curvature used in the proof.

In [FW1], Ferry and Weinberger extended the Farrell-Hsiang argument to include the noncompact case. This recovers Kasparov's theorem and extends it to include the prime 2. We shall sketch a proof of the Novikov Conjecture for closed Riemannian manifolds of nonpositive curvature which blends elements of the Farrell-Hsiang approach with elements of the argument from [FW1]. One pleasant aspect of this argument is that it shows that if $f: N \rightarrow M$ is a homotopy equivalence from a closed $n$-manifold $N$ to a closed nonpositively curved $n$-manifold $M$, then $f$ is covered by an unstable equivalence of tangent bundles. This unstable equivalence was used in [FW1] to show that the $A$-theory assembly map (see the section on $K$-theory above)

$$
W_{+} \wedge A(*) \rightarrow A(W)
$$

also splits for $W$ a complete Riemannian manifold of nonpositive curvature.

Let $M^{n}$ be a closed Riemannian manifold with nonpositive curvature. To prove that the assembly map

$$
A: H_{n}\left(M ; \mathbb{L}_{\bullet}\right) \rightarrow L_{n}\left(\mathbb{Z}\left[\pi_{1}(M)\right]\right)
$$

is a monomorphism, it suffices to show that the map $\mathcal{S}^{T O P}(M) \rightarrow H_{n}\left(M ; \mathbb{L}_{\bullet}\right)$ is zero. Thus, if $f: N \rightarrow M$ is a homotopy equivalence, we need to show that $f$ is normally cobordant to the identity. That is, we must show that there is a cobordism $(W, N, M)$ and a map $F: W \rightarrow M \times I$ so that $\left.F\right|_{N}=f$, $\left.F\right|_{M}=i d$, and so that $F$ is covered by a map of stable normal bundles.

Let $\tilde{f}: \widetilde{N} \rightarrow \widetilde{M}$ be the induced map on universal covers and form the 
diagram:

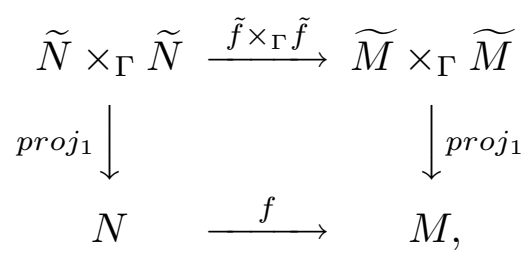

where $\Gamma=\pi_{1}(M)=\pi_{1}(N)$ acts diagonally on the product spaces. The fiberpreserving map $\tilde{f} \times_{\Gamma} \tilde{f}$ restricts to $\tilde{f}$ on the fibers of the bundle projections $\operatorname{proj}_{1}$.

Since $M$ is nonpositively curved, $\widetilde{M}$ has a natural compactification to a disk, $\bar{M} \cong D^{n}$. The action of $\Gamma$ on $\widetilde{M}$ extends to $\bar{M}$ and we obtain a disk bundle $\widetilde{M} \times_{\Gamma} \bar{M} \rightarrow M$. This compactification induces a similar compactification of $\widetilde{N}$ by adding an $(n-1)$-sphere at infinity. By the Černavskii-Seebeck theorem (see [Fer1]), the compactified fibers $\bar{N}$ are disks and an argument using local contractibility of the homeomorphism group shows that $\widetilde{N} \times_{\Gamma} \bar{N} \rightarrow N$ is a disk bundle. The induced map of boundaries is a homeomorphism on each fiber, so fiberwise application of the Alexander coning trick shows that $\tilde{f} \times_{\Gamma} \tilde{f}$ is homotopic to a fiber-preserving homeomorphism. Making this homotopy transverse to the zero section $s(m)=[\tilde{m}, \tilde{m}]$, where $m \in M$ and $\tilde{m}$ is any lift of $m$ to $\widetilde{M}$, gives the desired normal cobordism. The twisted products $\widetilde{M} \times_{\Gamma} \widetilde{M}$ and $\widetilde{N} \times_{\Gamma} \widetilde{N}$ contain copies of the tangent microbundle so, by Kister's theorem [Kis], they are isomorphic to the tangent microbundles of $M$ and $N$ and unstable tangentiality follows. For details, see [FW1].

We shall now show that the above assembly map is split. As we have seen in the section on surgery spectra (Quinn) the surgery exact sequence is the long exact homotopy sequence of a (co)fibration of spectra:

$$
\mathbb{H} \bullet\left(M ; \mathbb{L}_{\bullet}\right) \stackrel{A}{\rightarrow} \mathbb{L}_{\bullet}\left(\mathbb{Z}\left[\pi_{1}(M)\right]\right) \rightarrow \mathbb{S}_{\bullet}(M)
$$

A spectrum-level version of the vanishing result above shows that the map $\mathbb{S}_{\bullet}(M) \rightarrow \Sigma \mathbb{H}_{\bullet}\left(M ; \mathbb{L}_{\bullet}\right)$ is nullhomotopic. Since

$$
\mathbb{L}_{\bullet}\left(\mathbb{Z}\left[\pi_{1}(M)\right]\right) \rightarrow \mathbb{S}_{\bullet}(M) \rightarrow \Sigma \mathbb{H} \bullet\left(M ; \mathbb{L}_{\bullet}\right)
$$

is also a (co)fibration sequence, the nullhomotopy allows us to lift a map homotopic to the identity map $\mathbb{S}_{\bullet}(M) \rightarrow \mathbb{S}_{\bullet}(M)$ to $\mathbb{L} \bullet\left(\mathbb{Z}\left[\pi_{1}(M)\right]\right)$, splitting the homotopy sequence of the (co)fibration. It follows that the assembly map is split injective.

We begin our discussion of the extension to complete Riemannian manifolds of nonpositive curvature by stating a noncompact Borel Conjecture, which applies to discrete groups $\Gamma$ which need not be finitely generated. 
Noncompact Borel Conjecture for $\Gamma$. Let $(M, \partial M)$ be an open $n$ dimensional noncompact manifold with boundary such that $M$ is aspherical and $\pi_{1}(M)=\Gamma$. Let $f: N^{n} \rightarrow M^{n}$ be a proper homotopy equivalence which is a homeomorphism on the union of $\partial N$ with a neighborhood of infinity. Then $f$ is homotopic to a homeomorphism relative to the boundary and relative to a (possibly smaller) neighborhood of infinity.

To see how this is related to the Borel Conjecture consider the surgery exact sequence

$$
\begin{aligned}
\cdots \rightarrow \mathcal{S}^{T O P} & (M \text { rel } \partial M \cup\{\text { nbhd. of infinity }\}) \\
& \rightarrow[M, \partial M \cup\{\text { nbhd. of infinity }\} ; G / T O P, *] \rightarrow L_{n}\left(\mathbb{Z}\left[\pi_{1}(M)\right]\right) .
\end{aligned}
$$

The middle term is the cohomology of $M$ rel $\partial M$ with compact supports and coefficients in $G / T O P$, so that

$$
\begin{aligned}
& {[M, \partial M \cup\{\text { nbhd. of infinity }\} ; G / T O P, *]} \\
& \qquad H_{c}^{0}\left(M, \partial M ; \mathbb{L}_{\bullet}\right) \cong H_{n}\left(M ; \mathbb{L}_{\bullet}\right) \cong H_{n}\left(B \Gamma ; \mathbb{L}_{\bullet}\right) .
\end{aligned}
$$

As in the compact case considered in the section on " $K$-theory analogues" we have :

Proposition. The Noncompact Borel Conjecture holds for a group $\Gamma$ if and only if the algebraic surgery assembly map $A_{\Gamma}: H_{*}\left(B \Gamma ; \mathbb{L}_{\bullet}(\mathbb{Z})\right) \rightarrow L_{*}(\mathbb{Z}[\Gamma])$ is an isomorphism and $\mathrm{Wh}(\Gamma)=0$.

Here is an analogous Novikov-type result for complete Riemannian manifolds of nonpositive curvature.

Theorem ([FW1]). Let $W$ be a complete Riemannian manifold of nonpositive curvature and dimension $\geq 4$. Suppose that $f: W \rightarrow W^{\prime}$ is a proper homotopy equivalence and a homeomorphism on the complement of some compact set. Then $f$ is canonically covered by an isomorphism of unstable tangent bundles. The isomorphism produced agrees with the isomorphism given by $f$ outside of a, perhaps larger, compact set.

Instead of compactifying and coning, the proof uses the Chapman-Ferry $\alpha$-approximation theorem, a rescaling argument, and local contractibility of the homeomorphism group to produce a tangent bundle isomorphism covering $f$. The advantage is that this construction only sees a finite neighborhood of the zero-section, so the bundle isomorphism automatically becomes the given isomorphism near infinity. 


\section{The Farrell-Jones program}

In this section, we shall outline the work of Farrell-Jones on topological rigidity and the Borel Conjecture. This work was a direct continuation of the work of Farrell-Hsiang discussed above. By the Proposition in the section on " $K$-theory analogues," the Borel Conjecture holds for a discrete group $\Gamma$ if and only if $\mathrm{Wh}(\Gamma)=0$ and the algebraic surgery assembly map $A$ : $H_{*}\left(B \Gamma ; \mathbb{L}_{\bullet}\right) \rightarrow L_{*}(\mathbb{Z}[\Gamma])$ is an isomorphism.

In [FarHs7], Farrell and Hsiang proved the Borel Conjecture in case $B \Gamma=M^{n}, n>4$, is a flat Riemannian manifold with odd order holonomy group. The argument is an interesting combination of geometry and algebra. By an argument of Epstein-Shub, the manifold $M$ supports expanding endomorphisms. It follows that given $\epsilon>0$ and a homotopy equivalence $f: N \rightarrow M$, a map $e: M \rightarrow M$ can be chosen so that the pullback $f^{e}$ of $f$ over $e$ is an $\epsilon$-controlled homotopy equivalence over (the upper copy of) $M$ in the sense of $[\mathrm{ChF}]$ :

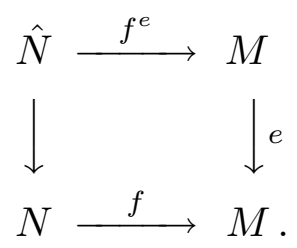

It follows from the " $\alpha$-Approximation" theorem of Chapman-Ferry quoted above that for small $\epsilon, f^{e}$ is homotopic to a homeomorphism. Farrell and Hsiang then use Frobenius induction to analyze the surgery exact sequence and show that the structure given by the original $f$ is trivial. The idea is that passage to finite covers corresponds to an algebraic transfer map and that if enough transfers of a structure are trivial, then the original structure is trivial, as well.

In [FJ5], Farrell and Jones proved the conjecture for closed hyperbolic manifolds. As in the work of Farrell-Hsiang, the idea is first to use differential geometry and a transfer argument to show that the transfer of an obstruction dies, and then to use an algebraic argument to deduce that the original obstruction is also zero.

In this case, the relevant geometry is the geometry of the geodesic flow on the unit sphere bundle of a hyperbolic manifold $M^{n}$. This flow is Anosov, which means that the sphere bundle admits a pair of transverse foliations such that the flow is expanding along one foliation and contracting along the other. Farrell and Jones show that it is possible to lift a homotopy equivalence $f: N \rightarrow M$ in such a way that the tracks of the lifted homotopies are pushed close to flow lines by the geodesic flow. This is the asymptotic transfer. Farrell and Jones use this construction to generalize results of Chapman 
and Ferry [ChF] and Quinn [Q3] to obtain a foliated control theorem. A full discussion of their results would occupy too much space for this survey, but we include a few precise statements to give the flavor of their work.

Definition. A path $\gamma$ in the unit sphere bundle $S M$ is said to be $(\beta, \epsilon)$ controlled if there is a second path $\phi$ in $S M$ such that

(1) The image of $\phi$ is contained in an arc of length $\beta$ inside a flow line of the geodesic flow.

(2) $d(\gamma(t), \phi(t))<\epsilon$ for all $t \in[0,1]$.

An $h$-cobordism $(W, S M)$ is said to be $(\beta, \epsilon)$-controlled if the tracks of the strong deformation retractions are $(\beta, \epsilon)$-controlled when pushed into $S M$.

Theorem. Given a closed hyperbolic manifold $M$ with $\operatorname{dim} M>2$ and a positive real number $\beta$, there exists a number $\epsilon>0$ such that the following is true. Every $(\beta, \epsilon)$-controlled $h$-cobordism $(W, S M)$ is a product.

The key property of the asymptotic transfer is that given positive real numbers $\beta$ and $\epsilon$ there is a positive number $t_{0}$ so that if $\alpha$ is a smooth path in $M$ of length $<\beta$, then the asymptotic lift $\bar{\alpha}$ of $\alpha$ to $S M$ becomes $(\sqrt{(} 2) \beta, \epsilon)$-controlled if we let the geodesic flow act on $\bar{\alpha}$ for any time $t \geq t_{0}$.

Together, these results show that if $(W, M)$ is an $h$-cobordism on $M$, then its transfer to the unit sphere bundle is a trivial $h$-cobordism. This is a key tool in their proof of topological rigidity for closed hyperbolic manifolds. We should emphasize that by itself this does not prove very much. Even though the fundamental groups of $M$ and $S M$ are isomorphic, the transfer map on the Whitehead groups is multiplication by 2 for $n$ odd and multiplication by 0 for $n$ even. Part of the solution is to modify this transfer to get a transfer to a disk bundle. Here, the transfer is multiplication by 1 and the analogous foliated control theorem shows that the Whitehead group of $M$ vanishes. Further effort is required to achieve topological rigidity. See [FJ3], [FJ5], [FJ7] for details.

In [FJ6], Farrell and Jones announced a proof of topological rigidity for closed nonpositively curved manifolds. The proof of this result is similar in outline, but much more complicated in execution. They begin by showing in [FJ10] that the Whitehead groups of the fundamental groups of these manifolds vanish. Given this, it suffices to show that if $f: N \rightarrow M$ is a homotopy equivalence then $f \times i d: M \times S^{1} \rightarrow N \times S^{1}$ is homotopic to a homeomorphism. Instead of transferring to the unit sphere bundle of $M$, they then transfer to a certain bundle over $M \times S^{1}$ whose fiber is a stratified space with three strata. The asymptotic transfer, which does nothing in the nonpositively curved case, is replaced by a focal transfer. See [FJ13] for 
details. The work of Farrell and Jones has also given significant results on integral versions of the Novikov Conjecture for algebraic $K$-theory [FJ12]; cf. the section on $K$-theory above.

\section{Problems about group actions (Weinberger et al.)}

Weinberger ([W3]-[W6]) has pointed out a number of interesting connections between the Novikov Conjecture and group actions. Aside from being interesting in itself, this work, together with the structure of the proofs of various versions of the Novikov Conjecture, has led to a study of "equivariant" versions of the Novikov Conjecture for manifolds equipped with group actions, a subject which is still under active development.

In general, equivariant surgery theory is considerably more complicated than ordinary surgery theory, both for geometric reasons (a manifold $M$ equipped with an action of, say, a compact group $G$ is stratified according to the various orbit types, and the relations between the various strata can be quite complicated) and for algebraic ones (in general, $G$ does not act on the fundamental group of $M$, but only on the fundamental groupoid; also, the algebra required to keep track of all the strata can be quite complicated). Weinberger in [W3]-[W5] studied the case where the algebra is as simple as possible, namely the case of "homologically trivial" actions, and found (this should not be so surprising, after all) that when $M$ is not simply connected, the theory of such actions is closely linked to ordinary non-simply connected surgery. As a result, he was able to prove the following:

Theorem [W4]. Let $G$ be a non-trivial finite group. Then the Novikov Conjecture is equivalent to the statement that the higher signatures vanish for any connected oriented manifold $M$ cobordant (by a bordism preserving the fundamental group) to a manifold $N$ admitting a free homologically trivial $G$-action (i.e, to a manifold $N$ admitting a free $G$-action, such that $\pi_{1}(N / G) \cong \pi_{1}(N) \times G$, and such that $G$ operates trivially on the homology of $N$ with local coefficients).

Theorem [W5]. Let $p$ be a prime, $G=\mathbb{Z} /(p)$. Suppose $G$ acts on a connected oriented manifold $M$ with non-empty connected fixed set $F$, with trivial action on $\Gamma=\pi_{1}(M)$ (computed at a basepoint in $F$ ) and on the homology of $M$ with local coefficients. (Also assume $G$ preserves the orientation if $p=2$.) By the $G$-signature theorem (the equivariant version of the Hirzebruch signature theorem), there is a rational characteristic class $\mathcal{D}$ of the equivariant normal bundle $\nu$ of $F$, such that

$$
\operatorname{signature}(M)=\langle\mathcal{L}(F) \cup \mathcal{D}(\nu),[F]\rangle \in \mathbb{Z}
$$


Then the Novikov Conjecture is equivalent to the statement that, in this context, the higher signatures of $M$ agree with the higher signatures of $F$ twisted by $\mathcal{D}(\nu)$, i.e., that if $u: M \rightarrow B \Gamma$ is the classifying map for the universal cover of $M$, then

$$
\operatorname{signature}_{x}(M, u)=\left\langle\mathcal{L}(F) \cup \mathcal{D}(\nu) \cup u^{*}(x),[F]\right\rangle
$$

for each cohomology class $x \in H^{*}(B \Gamma ; \mathbb{Q})$.

Subsequent work by Weinberger, discussed in detail in his book [W9], deals with applications and analogues of the Novikov Conjecture in much more complicated situations involving stratified spaces or manifolds with group actions. We shall discuss just one aspect of this here, the concept of the equivariant Novikov Conjecture, which is considered in [FRW], [RW1][RW3], and [Gong]. Using Kasparov's notion of the $K$-homology class $\left[D_{M}\right]$ of the signature operator $D_{M}$ on a closed oriented manifold $M$, and following [RW2], we may explain this as follows. Suppose $f: N \rightarrow M$ is an orientationpreserving homotopy equivalence of closed manifolds. The ordinary Novikov Conjecture says that if $X$ is an aspherical space and $u: M \rightarrow X,{ }^{16}$ then

$$
u_{*}\left(\left[D_{M}\right]\right)=(u \circ f)_{*}\left(\left[D_{N}\right]\right)
$$

at least rationally. Now suppose that a compact group $G$ acts on $M$ and $N$ and that the map $f$ is also $G$-equivariant (though not necessarily an equivariant homotopy equivalence). A $G$-space $X$ is called equivariantly aspherical if for every subgroup $H$ of $G$ (including the trivial subgroup!), every connected component of the fixed set $X^{H}$ is aspherical. For example, it follows from the Cartan-Hadamard Theorem (see [RW2, Proposition 1.5]) that if $X$ is a complete Riemannian manifold of nonpositive curvature, and if $G$ acts on $X$ by isometries, then $X$ is equivariantly aspherical. The equivariant Novikov Conjecture asserts that for suitable equivariantly aspherical spaces $X$, where $u: M \rightarrow X$ is a $G$-map and $f: N \rightarrow M$ is as above, then again $u_{*}\left(\left[D_{M}\right]\right)=(u \circ f)_{*}\left(\left[D_{N}\right]\right)$, the equality holding in the equivariant $K$-homology $K_{*}^{G}(X)$. While this is definitely false in some cases, it seems plausible when $X$ satisfies some equivariant finiteness conditions, and various cases are proved in the references cited above.

\footnotetext{
${ }^{16}$ Any map will do here. Often $u$ is the classifying map for the universal cover, but this need not be so.
} 


\section{Work related to the Novikov Conjecture: The Last 12 Years or So, III: Elliptic Operators and Operator Alge- bras}

In the last decade and a half, the analytic approach to the Novikov Conjecture, first introduced by Lusztig, has led to an explosion of research on various problems concerning elliptic operators and operator algebras. While we can only hint at some of these developments, we shall at least try to give the reader some impression of the various directions in which the subject is moving.

\section{Further development of Kasparov $K K$-theory}

To understand any of the further analytic developments, one needs to examine some of the ideas behind the work of Mishchenko and Kasparov, and in particular, why the $K$-theory of group $C^{*}$-algebras plays such a critical role in analytic approaches to the Novikov Conjecture. We shall be very brief; for further details, see the survey [Ros6] elsewhere in this volume.

The following is the basic idea of the analytic approach. Consider, say, a closed manifold $M$ with fundamental group $\Gamma$, equipped with the classifying map $f: M \rightarrow B \Gamma$ for the universal cover. Without great loss of generality, suppose the dimension $n$ of $M$ is even, $n=2 k$. We compare a certain analytic invariant of $M$, which one can call the analytic higher signature, with an a priori homotopy invariant, the Mishchenko symmetric signature. The former is the generalized index of a certain (generalized) elliptic operator; it plays the role of the index of a family of twisted signature operators in Lusztig's proof. Recall that the index of a family of operators parameterized by a compact space $Y$ is a certain formal difference of vector bundles over $Y$, in other words an element of the Grothendieck group of vector bundles, $K^{0}(Y)$. By the Serre-Swan Theorem, vector bundles over $Y$ correspond precisely (via passage to the space of continuous sections) to finitely generated projective modules over the ring $C(Y)$ of continuous functions on $Y$, so that $K^{0}(Y)$ may be identified with $K_{0}(C(Y))$. In Lusztig's case, $\Gamma$ was free abelian, $Y$ is the Pontrjagin dual $\widehat{\Gamma}$, and by Fourier analysis, $C(Y)$ may be identified with a $C^{*}$-algebra completion of the group ring $\mathbb{C}[\Gamma]$. In a similar fashion, in the general case the analytic higher signature is a formal difference of finitely generated projective modules over the completed group ring $C^{*}(\Gamma)$, and thus takes its values in the $K$-group $K_{0}\left(C^{*}(\Gamma)\right.$ ). On the other hand, the Mishchenko symmetric signature lives in the Wall group $L_{2 k}\left(C^{*}(\Gamma)\right)=L_{0}\left(C^{*}(\Gamma)\right.$ ). (In this case the symmetric and quadratic $L$-groups coincide and are 2-periodic, since we are taking $C^{*}(\Gamma)$ to be an algebra over $\mathbb{C}$.) 
The key idea that makes it possible to compare the two invariants is an observation originally going back to Gelfand and Mishchenko [GM]: for complex $C^{*}$-algebras, unlike general rings with involution, the functors $L_{0}$ and $K_{0}$ are naturally isomorphic. (This is due to the Spectral Theorem for self-adjoint operators on a Hilbert space, which implies that any nonsingular hermitian form on a $C^{*}$-algebra can be split as a direct sum of a positive-definite form and a negative-definite form.) One can then show ([Kas4], [Kas8], [KM2]) that under this isomorphism, the analytic higher signature coincides with the Mishchenko symmetric signature, and is thus homotopy-invariant. On the other hand, one can prove an index theorem, which implies that the analytic higher signature is the image under a certain analytic assembly map

$$
A: K_{*}(B \Gamma) \rightarrow K_{*}\left(C^{*}(\Gamma)\right)
$$

of $f_{*}\left(\left[D_{M}\right]\right)$, where $\left[D_{M}\right]$ is the class of the Atiyah-Singer signature operator on $M$ in Kasparov's analytic $K$-homology group. Thus if $A$ is injective, $f_{*}\left(\left[D_{M}\right]\right)$, which under the Chern character corresponds (up to some powers of 2 ) to $f_{*}$ of the total $L$-class, is a homotopy invariant of $M$. In other words, the higher signatures of $M$ are homotopy invariants.

Thus, just as in the algebraic approaches to the Novikov Conjecture, the conjecture boils down to the injectivity of a certain assembly map $A$. Within the last decade or so, various strategies for proving this injectivity have been simplified and strengthened. The approach of Kasparov and his co-workers has basically been to construct a splitting $s$ to the assembly map using a "generalized elliptic operator" (some variant of "dual Dirac"), and to prove that $s \circ A=$ id using the $K K$-calculus. In fact, this has usually been done by showing that $A$ and $s$ come from equivariant $K K$-classes on the universal cover $E \Gamma$ of $B \Gamma$, and then applying the equivariant $K K$ calculus ([Kas4], [Kas7]). This program has by now been extended to quite a number of situations: discrete subgroups of Lie groups [Kas7], groups acting on buildings ([JuV], [KS1], [KS2]), hyperbolic or even "bolic" groups ([HilS], $[\mathrm{KS} 3])$, and so on.

\section{The cyclic homology approach (Connes et al.)}

One of the most important new developments has been the introduction by Connes (from the point of view of geometry and analysis, [Con1]-[Con7]) and by Loday-Quillen [LodQ] and Tsygan [Tsy] (from the point of view of algebra and topology) of cyclic homology and cohomology $\mathrm{HC}_{*}$ and $\mathrm{HC}^{*}$, homology and cohomology theories for algebras which, when specialized to the algebra $C^{\infty}(M)$ of smooth functions on a manifold, recover de Rham 
homology and cohomology theory, based on differential forms and currents. The fundamental perspective here is that cyclic homology should be viewed as a "linearized" version of $K$-theory, and bears the same relationship to the algebraic $K$-theory of general algebras that ordinary (co)homology bears to topological $K$-theory. Motivated by this relationship, one can construct a natural transformation, the Chern character, from $K$-theory to cyclic homology.

The idea of applying cyclic homology to the Novikov Conjecture is based on the hope of finding a completion $\mathcal{A}(\Gamma)$ of the group ring $\mathbb{C}[\Gamma]$ so that $\mathbb{C}[\Gamma] \subseteq \mathcal{A}(\Gamma) \subseteq C^{*}(\Gamma)^{17}$ and the following two properties hold:

(1) $\mathcal{A}(\Gamma)$ is "big enough" so that the inclusion $\mathcal{A}(\Gamma) \hookrightarrow C^{*}(\Gamma)$ induces an isomorphism on $K_{0}$, and

(2) $\mathcal{A}(\Gamma)$ is "small enough" so that the inclusion $\mathbb{C}[\Gamma] \hookrightarrow \mathcal{A}(\Gamma)$ induces an injective map on cyclic homology.

Now there is an analogue of the assembly map $A$ for cyclic homology, and in the case of the group ring $\mathbb{C}[\Gamma]$, it is quite easy to show this assembly map is a split injection (for any group $\Gamma$ ) [Bur]. On the other hand, as we mentioned above, the usual Novikov Conjecture is a consequence of injectivity of the assembly map for $K$-theory of the group $C^{*}$-algebra, because of the special relationship between $L$-theory and $K$-theory for $C^{*}$-algebras. (Knowledge of $K_{0}$ is all one needs to handle even-dimensional manifolds, and odd-dimensional manifolds can be handled by crossing with a circle and replacing $\Gamma$ by $\Gamma \times \mathbb{Z}$.) The idea of the cyclic homology approach is therefore to construct a commutative diagram

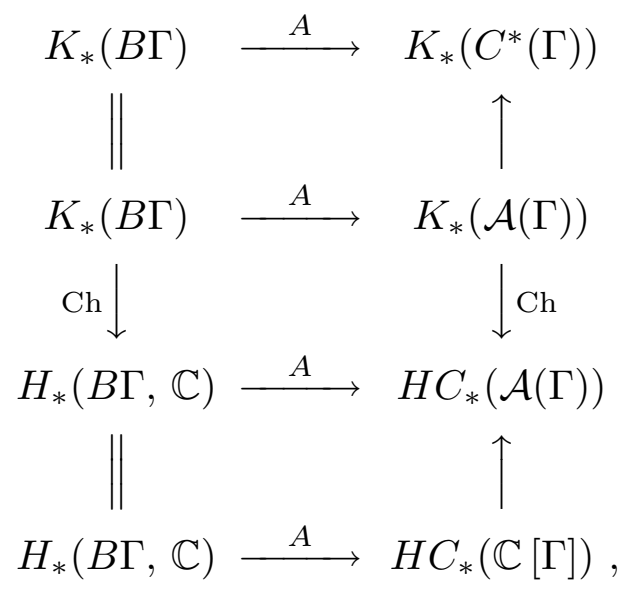

where the Chern character $\mathrm{Ch}$ induces an isomorphism $K_{*}(B \Gamma) \otimes_{\mathbb{Z}} \mathbb{C} \cong$

\footnotetext{
${ }^{17}$ The experts will realize that sometimes one needs to use the reduced group $C^{*}$ algebra here.
} 
$H_{*}(B \Gamma, \mathbb{C})$, and thus to deduce from (1) and (2) that the top assembly map is rationally injective.

In some cases, this program has been carried out successfully. As notable successes of the program, we mention in particular [CM1]-[CM2], [CGM2], [Jo1]-[Jo2], [Ji1]-[Ji3].

\section{$K$-theory of group $C^{*}$-algebras: the Connes-Kasparov and Baum- Connes Conjectures}

As we indicated above, the work of Mishchenko and Kasparov showed that the Novikov Conjecture for a group $\Gamma$ is a consequence of something stronger (now often called the "Strong Novikov Conjecture"), the injectivity of the assembly map $K_{*}(B \Gamma) \stackrel{A}{\longrightarrow} K_{*}\left(C^{*}(\Gamma)\right)$. Thus the study of the Novikov Conjecture naturally leads to the study of the $K$-theory of group $C^{*}$-algebras. This study has led in turn to a number of related conjectures, which have been verified in many cases.

To begin with, the classifying space $B G$ and the group $C^{*}$-algebra $C^{*}(G)$ are defined not only for discrete groups, but also for all locally compact groups $G$, and circumstantial evidence [Ros1] suggests a close connection between the $K$-theory of the classifying space and of the $C^{*}$-algebra for arbitrary Lie groups.

Secondly, it is useful to try to examine what sort of surjectivity one should expect for the assembly map. For non-amenable discrete groups $\Gamma$, the "full" $C^{*}$-algebra $C^{*}(\Gamma)$ turns out to be "too big" (in the sense that even for nice torsion-free groups, one cannot expect surjectivity), so it seems the appropriate object of study is the "reduced" $C^{*}$-algebra $C_{r}^{*}(\Gamma)$. This is a quotient of $C^{*}(\Gamma)$ for which one also has a functorial assembly map $A$. By extrapolating from carefully studied examples, Baum and Connes ([BC3], $[\mathrm{BC} 4],[\mathrm{BCH}])$ arrived at the conjecture that a certain modified assembly map

$$
A^{\prime}: K_{*}^{\Gamma}(\mathcal{E} \Gamma) \rightarrow K_{*}\left(C_{r}^{*}(\Gamma)\right)
$$

should be an isomorphism for any discrete group. Here $\mathcal{E} \Gamma$ is the universal proper $\Gamma$-space, just as $B \Gamma$ is the quotient of the universal free $\Gamma$-space $E \Gamma$ by the $\Gamma$-action. When $\Gamma$ is torsion-free, $\Gamma$ acts freely on $\mathcal{E} \Gamma=E \Gamma$, so the equivariant $K$-group on the left becomes just $K_{*}(B \Gamma)$, and $A^{\prime}=A$. Hence the Baum-Connes Conjecture predicts that for torsion-free groups, the assembly map of Mishchenko and Kasparov is an isomorphism if one uses the reduced $C^{*}$-algebra. In general, $A$ factors through $A^{\prime}$, and the natural map

$$
K_{*}(B \Gamma) \cong K_{*}^{\Gamma}(E \Gamma) \rightarrow K_{*}^{\Gamma}(\mathcal{E} \Gamma)
$$


is at least rationally injective, so the Baum-Connes Conjecture implies the Novikov Conjecture.

Various extensions and strengthenings of the Baum-Connes Conjecture have been proposed. For example, Baum and Connes [BCH] also suggested that if $\Gamma$ is replaced by any countably connected Lie group $G$, acting on a $C^{*}$-algebra $A$, then there should be a canonical assembly isomorphism from $K_{*}^{G}(\mathcal{E} G ; A)$ to $K_{*}\left(A \rtimes_{r} G\right)$. Here $A \rtimes_{r} G$, also sometimes written $C_{r}^{*}(G, A)$, is the reduced crossed product $C^{*}$-algebra. The previous conjecture is just the special case where $A=\mathbb{C}$ and $G$ is discrete. When $G$ is a connected Lie group with maximal compact subgroup $K$, then $\mathcal{E} G=G / K$, so if we take $A=\mathbb{C}$ again, the conjecture reduces to the assertion that the "Dirac induction" map

$$
\mu: R(K)=K_{*}^{K}(p t) \cong K_{*}^{G}(G / K) \rightarrow K_{*}\left(C_{r}^{*}(G)\right)
$$

(here $R(G)$ is the representation ring of $K$ ) should be an isomorphism. This special case of the generalized Baum-Connes Conjecture is usually known as the Connes-Kasparov Conjecture, and has been proved for connected linear reductive Lie groups by Wassermann [Was]. Similar results are known for some $p$-adic Lie groups (e.g., [Pl3], [BHP], [BCH]). For arbitrary closed subgroups $G$ of amenable connected Lie groups, of $S O(n, 1)$, or of $S U(n, 1)$, the generalized Baum-Connes Conjecture follows from still stronger results of Kasparov et al. ([Kas4], [Kas5], [Kas7], [JuK]).

\section{Parallels with positive scalar curvature (Gromov-Lawson, Rosen- berg et al.)}

Around 1980, Gromov and Lawson ([GL1], [GL2]) began to notice an interesting parallel between the Novikov Conjecture and a problem in Riemannian geometry, that of determining what smooth manifolds admit Riemannian metrics of positive scalar curvature. They conjectured in particular that a closed aspherical manifold could not admit such a metric, and that a general spin manifold (except perhaps in dimensions 3 and 4) should admit such a metric exactly when certain "higher index invariants" vanish. Originally, the parallel with the Novikov Conjecture was just phenomenological: both problems were related to index theory on non-simply connected manifolds, and in both cases the best results were for aspherical manifolds homotopy-equivalent to $K(\Gamma, 1)$ 's satisfying some sort of "non-positive curvature" condition.

In a series of papers [Ros2]-[Ros5], Rosenberg showed that this coincidence was not accidental, and that in fact both problems are closely related to the Strong Novikov Conjecture. The formal similarity between the two 
problems is explained via the index theory of Mishchenko and Fomenko [MisF], applied to the canonical flat bundle $\mathcal{V}=\widetilde{M} \times_{\Gamma} C^{*}(\Gamma)$ over a manifold $M$, whose fibers are rank-one free modules over the group $C^{*}$-algebra $C^{*}(\Gamma)$ of the fundamental group. The main differences between the two problems are that:

(1) the Novikov Conjecture is related to the signature operator, whereas the positive scalar curvature problem (on a spin manifold $M$ ) is related to the Dirac operator $\not_{M}$ via the Lichnerowicz identity $\not_{M}^{2}=\nabla^{*} \nabla+$ $\frac{s}{4}$, where $s$ is the scalar curvature;

(2) the Novikov Conjecture and the Borel Conjecture are most directly related to the assembly map for $L$-theory, whereas the positive scalar curvature problem is related to the assembly map for $K O$-theory. In particular, the two theories behave very differently at the prime 2 [Ros6].

Rosenberg gave [Ros5] a reformulation of the Gromov-Lawson Conjecture with some hope of being true for arbitrary spin manifolds, and he and Stolz (whose techniques have proven to be crucial for the problem) have now verified a "stable" form of the Gromov-Lawson Conjecture for a wide variety of fundamental groups, including in particular all finite groups [RS1]-[RS3].

\section{Analogues for foliations}

Around 1980, A. Connes [Con1] pointed out that one can attach operator algebras to foliations, and use them to develop index theory for operators associated with foliated manifolds: at first, operators which are "elliptic along the leaves," such as the Dirac and signature operators of the leaves [CS], and later, certain transversally elliptic operators (i.e., elliptic operators on the "leaf space") [Con2]. From these beginnings it was only a small step to the study of an assembly map

$$
A: K_{*}(B(M, \mathcal{F})) \rightarrow K_{*}\left(C_{r}^{*}(M, \mathcal{F})\right)
$$

[BC1] for foliated manifolds $(M, \mathcal{F})$ and to analogues for foliations of the Novikov, Borel, and Baum-Connes Conjectures. The first formulation of a Novikov Conjecture for foliations was given by Baum and Connes in [BC2]. The conjecture states that if $(M, \mathcal{F}),\left(M^{\prime}, \mathcal{F}^{\prime}\right)$ are foliated closed manifolds, with $M$ and $M^{\prime}$ compact and oriented and with $\mathcal{F}$ and $\mathcal{F}^{\prime}$ orientable, then if $f: M^{\prime} \rightarrow M$ is an orientation-preserving leafwise homotopy equivalence (i.e., leaf-preserving map which is a homotopy equivalence in a leaf-preserving way) and if $u: M \rightarrow B \pi$ is the canonical map to the classifying space of the fundamental groupoid of $(M, \mathcal{F})$ along the leaves, then for any $x \in$ $H^{*}(B \pi ; \mathbb{Q})$, the "higher signatures"

$$
\left\langle\mathcal{L}(M) \cup u^{*}(x),[M]\right\rangle \quad \text { and } \quad\left\langle\mathcal{L}\left(M^{\prime}\right) \cup(u \circ f)^{*}(x),\left[M^{\prime}\right]\right\rangle
$$


should be equal. As pointed out by Baum and Connes, when $M$ and $M^{\prime}$ each consist of a single leaf, this is the usual Novikov Conjecture, and when $M$ and $M^{\prime}$ are each foliated by points (i.e., $\mathcal{F}$ and $\mathcal{F}^{\prime}$ are zero-dimensional), this reduces to Novikov's theorem on the homeomorphism invariance of rational Pontrjagin classes. Using a variant of Kasparov's methods, Baum and Connes verified their Conjecture when there is a Riemannian metric on $M$ for which the sectional curvatures of the leaves are all non-positive. Other cases of the Novikov Conjecture for foliations and of the analogues of the Baum-Connes and Gromov-Lawson Conjectures for foliations have been settled in [Na2], [To], [Mac], [Tak1]-[Tak3], and [Hu4].

\section{Flat and almost flat bundles revisited (Connes-Gromov-Moscovici,} Gromov et al.)

A major theme in analytic work on the Novikov Conjecture, which already appeared in the pioneering work of Lusztig, has been the use of flat and almost flat bundles. These have also been used by Gromov [Gr6] within the last year to give a very slick proof of Novikov's theorem on the topological invariance of rational Pontrjagin classes. Since this is a little easier than the work on the Novikov Conjecture, we mention it first. As is well known, Novikov's theorem is essentially equivalent (see [Ran10, §2] for a few more details) to the statement that if $M^{4 k+r}$ is a closed oriented manifold and $N^{4 k}$ is the inverse image of a regular value of a map $M \rightarrow S^{r}$, then the signature of $N$ is a homeomorphism invariant of $M$. Since homeomorphisms restrict on open subsets to proper homotopy equivalences, it is then enough to show that signature $(N)$ only depends on the proper homotopy type of some open tubular neighborhood $U$. One can reduce to the case where $r=2 m+1$ is odd, and then embed in $S^{r}$ a product $\left(\Sigma^{2}\right)^{m} \times \mathbb{R}$, where $\Sigma^{2}$ is a closed Riemann surface of genus $>1$. (That such an embedding is possible follows from the fact that $\Sigma$ is stably parallelizable.) Gromov then uses the fact that $\Sigma$ admits a flat rank-2 real vector bundle $X$ with structure group $S L(2, \mathbb{R})$ and non-trivial Euler class. Since $S L(2, \mathbb{R}) \cong S p\left(\mathbb{R}^{2}\right)$, this bundle comes with a natural symplectic structure, which gives rise (because of anti-symmetry of the cup product for odd cohomology classes) to a non-degenerate symmetric bilinear form on the cohomology with local coefficients, $H^{1}(\Sigma ; X)$. One can compute that the signature $\sigma$ of this form turns out to be non-zero. Putting $m$ copies of $X$ together and pulling back to $U$, Gromov obtains a twisted signature invariant for $U$ that can be shown (using the original ideas of Novikov) to be a proper homotopy invariant, but which differs from signature $(N)$ only by a non-zero constant factor $\left(\sigma^{m}\right)$, and so Novikov's theorem follows. See [Ran10, §4] for the surgery-theoretic interpretations of Novikov's and Gromov's proofs of the topological invariance of the rational Pontrjagin 
classes.

The application of flat bundles to the Novikov Conjecture similarly originates from the rather simple observation that if $M^{4 k}$ is a closed oriented manifold and $X$ is a flat vector bundle over $M$ with structure group $O(m)$ or $U(m)$, then one can define a symmetric or hermitian pairing on the cohomology with local coefficients, $H^{2 k}(M ; X)$, and thus a "twisted signature" signature $_{X}(M)$. It is clear that this signature is an oriented homotopy invariant. It is also not hard to show that signature $_{X}(M)$ coincides with the higher signature signature ${ }_{x}(M, u)$, where (since $X$ is flat) the Chern character $x$ of $X$ is the pull-back under some $u: M \rightarrow B \Gamma$ of a cohomology class $x \in H^{*}(B \Gamma ; \mathbb{Q})$. The only difficulty is that since flat vector bundles with structure group $O(m)$ or $U(m)$ have trivial rational characteristic classes, all one gets from this argument is the homotopy invariance of the usual signature.

Nevertheless, there are several ways of getting around this difficulty to use this argument to prove results on the Novikov Conjecture. One idea (in effect the idea of the Mishchenko and Kasparov methods) is to replace the ordinary vector bundle $X$ by a flat bundle with infinite-dimensional fibers (which are finitely generated projective modules over $C^{*}(\Gamma)$ ). Another possibility, explored by Lusztig [Lus] and Gromov [Gr6], is to use indefinite orthogonal or unitary groups in place of $O(m)$ or $U(m)$. But an alternative is to use ordinary finite-dimensional bundles, but which are only "approximately" flat, yet which come from representations of the fundamental group. It is this approach which is adopted in [CGM1] and [HilS], and also discussed in [Gr6]. Here the idea is roughly that since the twisted signature (i.e., the index of the signature operator with coefficients in a bundle) is a "discrete" invariant, "small" amounts of non-flatness do not affect that argument that this is a homotopy invariant.

\section{Index theory on non-compact manifolds (Roe et al.)}

The original ideas of Novikov for proving topological invariance of rational Pontrjagin classes made essential use of non-compact manifolds, so it is not surprising that analysis on such manifolds is starting to play a bigger and bigger role in recent work on the Novikov Conjecture. While it would be impossible to survey here everything that has been done using analysis on non-compact manifolds that is related to the Novikov Conjecture, we shall mention a few key themes, especially as found in the work of J. Roe and his coworkers. Much of the impetus for this work came from an important paper of Atiyah [At2], which showed how the theory of operator algebras could be used to study index theory on the universal covers of compact manifolds. To illustrate how the theory works, we shall begin by discussing 
some results on the Gromov-Lawson Conjecture (see the section above on positive scalar curvature). The index theory of the Dirac operator $D_{M}$ shows via the Lichnerowicz identity that if $M$ is a closed spin manifold with $\hat{A}$-genus

$$
\hat{A}(M)=\langle\hat{\mathcal{A}}(M), M\rangle \neq 0
$$

(here $\hat{\mathcal{A}}(M)$ is a certain formal power series in the rational Pontrjagin classes of $M$ ), then $M$ does not admit a metric of positive scalar curvature. (Positive scalar curvature would imply $\not_{M}$ is invertible by the Lichnerowicz identity, whereas the index of $\not_{M}$ is $\hat{A}(M) \neq 0$ by the Atiyah-Singer Theorem.) If $M$ has fundamental group $\Gamma$ and universal cover $\widetilde{M}$, then one could similarly argue using Atiyah's index theorem [At2] that $\widetilde{M}$ does not admit a $\Gamma$-invariant metric of positive scalar curvature. However, this argument does not yet exploit the extra "flexibility" of non-compact manifolds as compared with closed manifolds, since most metrics on $\widetilde{M}$ are not $\Gamma$-invariant. A more powerful result, proved by Roe [Roe1] using a more robust version of index theory on non-compact manifolds, is that under these circumstances, $\widetilde{M}$ does not admit any metric, quasi-isometric to a $\Gamma$-invariant metric, whose scalar curvature function is uniformly positive off a compact set.

Once the technology for proving this result was in place, it became possible to prove that certain aspherical manifolds $M$ (such as tori, which have vanishing $\hat{A}$-genus) do not admit Riemannian metrics of positive scalar curvature, by showing that $\widetilde{M}$ does not admit any metric of uniformly positive scalar curvature in the appropriate quasi-isometry class. It is here that "coarse geometry" and "coarse homology" (also known as "exotic homology," though this is less descriptive) come into play. The Atiyah-Singer Theorem on a compact manifold computes the index of an elliptic operator, which is a certain obstruction to its non-invertibility, as a certain characteristic cohomology class (determined by the symbol of the operator) paired against the fundamental class in homology. Roe's index theory in [Roe3] does something similar for elliptic operators on non-compact manifolds, but now one is only interested in the asymptotic behavior of the manifold at infinity, and compact sets can be thrown away. One still finds that there are homological obstructions to the non-invertibility of the operator, but they involve the homology of the manifold "at infinity." This may be made precise either in terms of the concept of a "corona" (introduced in [Hig2] as a prototypical example, the sphere at infinity $S^{n-1}$ is a corona for the Euclidean space $\mathbb{R}^{n}$ ) or in terms of Roe's coarse homology theory [Roe3].

Corresponding to these results on the Gromov-Lawson Conjecture, there are also results on the Novikov Conjecture itself using the machinery of 
"coarse homology." For more details, we refer the reader to the paper [HigR2] in these proceedings. However, the main idea can be stated briefly as follows. For any "proper metric space" $X,{ }^{18}$ one can define a coarse assembly map

$$
A^{c}: K_{*}^{\ell f}(X) \rightarrow K_{*}\left(C^{*}(X)\right) .
$$

Here $K_{*}^{\ell f}(X)$ is the locally finite $K$-homology of $X$, in other words, the reduced Steenrod $K$-homology of the one-point compactification [Fer2], and $K_{*}\left(C^{*}(X)\right)$ is the topological $K$-theory of the $C^{*}$-algebra constructed in [Roe3] out of "generalized pseudodifferential operators" on $X$. This assembly map factors through the "coarse $K$-homology" $K X_{*}(X)$. In favorable cases, one can prove injectivity of this coarse assembly map $A^{c}$ with $X=E \Gamma$, and then deduce from a commutative diagram the injectivity of the usual assembly map

$$
A: K_{*}(B \Gamma) \rightarrow C_{r}^{*}(\Gamma)
$$

in other words, the "strong Novikov Conjecture" for $\Gamma$. (See [HigR2], [Yu3][Yu5], [FW2], [PeRW], and [Ros6] for more information.)

\section{REFERENCES}

[Ad] A. Adem, Characters and K-theory of discrete groups, Invent. Math. 114 (1993), 489-514.

[AnCFK] D. R. Anderson, F. X. Connolly, S. C. Ferry, and E. K. Pedersen, Algebraic $K$-theory with continuous control at infinity, J. Pure Appl. Alg. 94 (1994), 25-48.

[AnM1] D. R. Anderson and H. J. Munkholm, Boundedly Controlled Topology, Lect. Notes in Math., vol. 1323, Springer-Verlag, Berlin, Heidelberg, New York, 1988.

[AnM2] D. R. Anderson and H. J. Munkholm, Geometric modules and algebraic K-homology theory, K-Theory 3 (1990), 561-602.

[Ang] N. Anghel, On the index of Callias-type operators, Geom. and Funct. Anal. 3 (1993), 431-438.

[At1] M. Atiyah, Characters and cohomology of finite groups, Publ. Math. Inst. Hautes Études Sci. 9 (1961), 23-64.

[At2] M. Atiyah, Elliptic operators, discrete groups and von Neumann algebras, Colloque analyse et topologie en l'honneur de Henri Cartan, Astérisque, vol. 32-33, Soc. Math. de France, Paris, 1976, pp. 43-72.

[Att1] O. Attie, Quasi-isometry classification of some manifolds of bounded geometry, Math. Z. 216 (1994), 501-527.

[Att2] O. Attie, A surgery theory for manifolds of bounded geometry, preprint, McMaster Univ. (1994).

\footnotetext{
${ }^{18}$ In the case of present interest, $X$ is a non-compact manifold, equipped with a complete Riemannian metric - for example, the universal cover $\widetilde{M}$ of a compact manifold $M$, equipped with the pull-back of a Riemannian metric on $M$.
} 
[AtBW] O. Attie, J. Block, and S. Weinberger, Characteristic classes and distortion of diffeomorphisms, J. Amer. Math. Soc. 5 (1992), 919-921.

[AsV] A. H. Assadi and P. Vogel, Semifree finite group actions on compact manifolds, Algebraic and geometric topology (New Brunswick, NJ, 1983), Lect. Notes in Math., vol. 1126, Springer-Verlag, Berlin, Heidelberg, New York, 1985, pp. $1-21$.

[Bass] H. Bass, Algebraic K-Theory, W. A. Benjamin, New York and Amsterdam, 1968.

[BHS] H. Bass, A. Heller and R. Swan, The Whitehead group of a polynomial extension, Publ. Math. Inst. Hautes Études Sci. 22 (1964), 61-79.

[BC1] P. Baum and A. Connes, Geometric K-theory for Lie groups and foliations, Preprint, Inst. Hautes Études Sci., 1982.

[BC2] P. Baum and A. Connes, Leafwise homotopy equivalence and rational Pontrjagin classes, Foliations: Proceedings, Tokyo, 1983 (I. Tamura, ed.), Advanced Studies in Pure Math., vol. 5, Kinokuniya and North-Holland, Tokyo, Amsterdam, 1985, pp. 1-14.

[BC3] P. Baum and A. Connes, Chern character for for discrete groups, A fête of topology, Academic Press, Boston, 1988, pp. 163-232.

[BC4] P. Baum and A. Connes, K-theory for discrete groups, Operator algebras and applications, Vol. 1 (D. Evans and M. Takesaki, eds.), London Math. Soc. Lecture Note Ser., vol. 135, Cambridge Univ. Press, Cambridge, 1988, pp. 1-20.

[BCH] P. Baum, A. Connes, and N. Higson, Classifying space for proper actions and $K$-theory of group $C^{*}$-algebras, $C^{*}$-algebras: 1943-1993, A Fifty-Year Celebration (R. S. Doran, ed.), Contemp. Math., vol. 167, Amer. Math. Soc., Providence, RI, 1994, pp. 241-291.

[BHP] P. Baum, N. Higson, and R. Plymen, Equivariant homology for SL(2) of a p-adic field, Index Theory and Operator Algebras (J. Fox and P. Haskell, eds.), Contemp. Math., vol. 148, Amer. Math. Soc., Providence, RI, 1993, pp. 1-18.

[BeM] S. Bentzen and I. Madsen, Trace maps in algebraic K-theory and the CoatesWiles homomorphism, J. reine angew. Math. 411 (1990), 171-195.

[Bi] R. Bieri, A group with torsion-free 2-divisible homology and Cappell's result on the Novikov Conjecture, Invent. Math. 33 (1976), 181-184.

[Black] B. Blackadar, K-Theory for Operator Algebras, Math. Sciences Research Inst. Publications, vol. 5, Springer-Verlag, New York, Berlin, Heidelberg, London, Paris, Tokyo, 1986.

[BlocW] J. Block and S. Weinberger, Aperiodic tilings, positive scalar curvature and amenability of spaces, J. Amer. Math. Soc. 5 (1992), 907-918.

[BöHM1] M. Bökstedt, W. C. Hsiang, and I. Madsen, The cyclotomic trace and the $K$-theoretic analogue of Novikov's conjecture, Proc. Nat. Acad. Sci. U.S.A. 86 (1989), 8607-8609.

[BöHM2] M. Bökstedt, W. C. Hsiang, and I. Madsen, The cyclotomic trace and algebraic K-theory of spaces, Invent. Math. 111 (1993), 465-539.

[Br1] W. Browder, Homotopy type of differentiable manifolds, Proc. Århus Topology Symp., unpublished mimeographed notes, 1962, reprinted in these Proceedings.

[Br2] W. Browder, Structures on $M \times \mathbb{R}$, Proc. Camb. Phil. Soc. 61 (1965), 337-345.

[Br3] W. Browder, Manifolds with $\pi_{1}=\mathbb{Z}$, Bull. Amer. Math. Soc. 72 (1966), $238-244$.

[Br4] W. Browder, Surgery on Simply-Connected Manifolds, Ergebnisse der Math. und ihrer Grenzgebiete, vol. 65, 1972. 
[BrHs] W. Browder and W. C. Hsiang, G-actions and the fundamental group, Invent. Math. 65 (1982), 411-424.

[BryF] J. Bryant, S. Ferry, W. Mio, and S. Weinberger, Topology of homology manifolds, Bull. Amer. Math. Soc. 28 (1993), 324-328.

[BryP] J. L. Bryant and M. E. Petty, Splitting manifolds as $M \times \mathbb{R}$ where $M$ has a $k$-fold end structure, Topology Appl. 14 (1982), 87-104.

[Bur] D. Burghelea, The cyclic cohomology of group rings, Comment. Math. Helv. 60 (1985), 354-365.

[Cap1] S. E. Cappell, A splitting theorem for manifolds and surgery groups, Bull. Amer. Math. Soc. 77 (1971), 281-286.

[Cap2] S. E. Cappell, A splitting theorem for manifolds, Invent. Math. 33 (1976), 69-170.

[Cap3] S. E. Cappell, On homotopy invariance of higher signatures, Invent. Math. 33 (1976), 171-179.

[CaSW] S. E. Cappell, J. L. Shaneson, and S. Weinberger, Classes topologiques caractéristiques pour les actions de groupes sur les espaces singuliers, C. R. Acad. Sci. Paris Sér. I Math. 313 (1991), 293-295.

[CaW1] S. E. Cappell and S. Weinberger, A geometric interpretation of Siebenmann's periodicity phenomenon, Geometry and topology (Athens, Ga. 1985), Lecture Notes in Pure and Appl. Math., vol. 105, Dekker, New York, NY, 1987, pp. $47-52$.

[CaW2] S. E. Cappell and S. Weinberger, Classification de certains espaces stratifiés, C. R. Acad. Sci. Paris Sér. I Math. 313 (1991), 399-401.

[Car1] G. Carlsson, Equivariant stable homotopy and Segal's Burnside ring conjecture, Ann. of Math. 120 (1984), 189-224.

[Car2] G. Carlsson, Segal's Burnside ring conjecture and the homotopy limit problem, Homotopy Theory (E. Rees and J. D. S. Jones, eds.), London Math. Soc. Lect. Notes, vol. 117, Cambridge Univ. Press, Cambridge, 1987, pp. 6-34.

[Car3] G. Carlsson, Homotopy fixed points in the algebraic K-theory of certain infinite discrete groups, Advances in Homotopy Theory (S. Salamon, B. Steer, and W. Sutherland, eds.), London Math. Soc. Lect. Notes, vol. 139, Cambridge Univ. Press, Cambridge, 1990, pp. 5-10.

[Car4] G. Carlsson, Proper homotopy theory and transfers for infinite groups, Algebraic Topology and its Applications (G. Carlsson, R. Cohen, W.-C. Hsiang, and J. D. S. Jones, eds.), M. S. R. I. Publications, vol. 27, Springer-Verlag, New York, 1994, pp. 1-14.

[Car5] G. Carlsson, Bounded K-theory and the assembly map in algebraic K-theory, $I$, these Proceedings.

[CarP] G. Carlsson and E. K. Pedersen, Controlled algebra and the Novikov conjectures for $K$ - and L-theory, Topology (to appear).

[Carter] D. W. Carter, Localization in lower algebraic K-theory, Comm. Algebra 8 (1980), 603-622; Lower K-theory of finite groups, Comm. Algebra 8 (1980), 1927-1937.

[ChapF] T. A. Chapman and S. C. Ferry, Approximating homotopy equivalences by homeomorphisms, Amer. J. Math. 101 (1979), 583-607.

[ChG] J. Cheeger and M. Gromov, Bounds on the von Neumann dimension of $L^{2}$-cohomology and the Gauss-Bonnet theorem for open manifolds, J. Diff. Geom. 21 (1985), 1-34.

[ChG] J. Cheeger and M. Gromov, $L^{2}$-cohomology and group cohomology, Topology 
[CoJ] R. L. Cohen and J. D. S. Jones, Algebraic K-theory of spaces and the Novikov conjecture, Topology 29 (1990), 317-344.

[Con1] A. Connes, A survey of foliations and operator algebras, Operator Algebras and Applications (Kingston, Ont., 1980) (R. V. Kadison, ed.), Proc. Symp. Pure Math., vol. 38, Part 1, Amer. Math. Soc., Providence, 1982, pp. 521-628.

[Con2] A. Connes, Cyclic cohomology and the transverse fundamental class of a foliation, Geometric methods in operator algebras (Kyoto, 1983) (H. Araki and E. G. Effros, eds.), Pitman Res. Notes Math., vol. 123, Longman Sci. Tech., Harlow, 1986, pp. 52-144.

[Con3] A. Connes, Introduction to noncommutative differential geometry, Arbeitstagung Bonn 1984, Lecture Notes in Math., vol. 1111, Springer-Verlag, Berlin, Heidelberg, New York, 1985, pp. 3-16.

[Con4] A. Connes, Cyclic cohomology and noncommutative differential geometry, Proc. International Congress of Mathematicians (Berkeley, Calif., 1986), vol. 1, Amer. Math. Soc., Providence, RI, 1987, pp. 879-889.

[Con5] A. Connes, Cyclic cohomology and noncommutative differential geometry, Géometrie différentielle (Paris, 1986), Travaux en Cours, vol. 33, Hermann, Paris, 1988, pp. 33-50.

[Con6] A. Connes, Géometrie non commutative, InterEditions, Paris, 1990.

[Con7] A. Connes, Noncommutative Geometry, Academic Press, San Diego, 1994.

[CGM1] A. Connes, M. Gromov, and H. Moscovici, Conjecture de Novikov et fibrés presques plats, C. R. Acad. Sci. Paris Sér. I Math. 310 (1990), 273-277.

[CGM2] A. Connes, M. Gromov, and H. Moscovici, Group cohomology with Lipschitz control and higher signatures, Geom. Funct. Anal. 3 (1993), 1-78.

[CM1] A. Connes and H. Moscovici, Conjecture de Novikov et groupes hyperboliques, C. R. Acad. Sci. Paris Sér. I Math. 307 (1988), 475-480.

[CM2] A. Connes and H. Moscovici, Cyclic cohomology, the Novikov conjecture and hyperbolic groups, Topology 29 (1990), 345-388.

[CS] A. Connes and G. Skandalis, The longitudinal index theorem for foliations, Publ. Res. Inst. Math. Sci. (Kyoto Univ.) 20 (1984), 1139-1183.

[CST] A. Connes, D. Sullivan and N. Teleman, Quasiconformal mappings, operators on Hilbert space, and local formula for characteristic classes, Topology $\mathbf{3 3}$ (1994), 663-681.

[CyK1] F. X. Connolly and T. Koźniewski, Finiteness properties of classifying spaces of proper $\Gamma$-actions, J. Pure Applied Algebra 41 (1986), 17-36.

[CyK2] F. X. Connolly and T. Koźniewski, Rigidity and crystallographic groups, I, Invent. Math. 99 (1990), 25-48.

[CyK3] F. X. Connolly and T. Koźniewski, Examples of lack of rigidity in crystallographic groups, Algebraic Topology, Poznań 1989 (S. Jackowski, B. Oliver, and K. Pawałowski, eds.), Lecture Notes in Math., vol. 1474, Springer-Verlag, Berlin, Heidelberg, and New York, 1991, pp. 139-145.

[Cu1] J. Cuntz, The K-groups for free products of $C^{*}$-algebras, Operator Algebras and Applications (Kingston, Ont, 1980) (R. V. Kadison, ed.), Proc. Symp. Pure Math., vol. 38, Part 1, Amer. Math. Soc., Providence, 1982, pp. 81-84.

[Cu2] J. Cuntz, K-theory for certain $C^{*}$-algebras, II, J. Operator Theory 5 (1981), 101-108.

[Da] M. W. Davis, Coxeter groups and aspherical manifolds, Algebraic topology, Aarhus 1982, Lecture Notes in Math., vol. 1051, Springer-Verlag, Berlin, Hei- 
delberg, New York, 1984, pp. 197-221.

[Di] Do Ngoc Diep, $C^{*}$-complexes de Fredholm, I, Acta Math. Vietnam. 9 (1984), 121-130; II, ibid. 9 (1984), 193-199.

[Do] R. G. Douglas, $C^{*}$-algebra extensions and K-homology, Ann. of Math. Studies, vol. 95, Princeton University Press, Princeton, NJ, 1980.

[DrFW] A. N. Dranishnikov, S. Ferry, and S. Weinberger, A large Riemannian manifold which is flexible, preprint, SUNY at Binghamton.

[Fack1] T. Fack, K-théorie bivariante de Kasparov, Séminaire Bourbaki, 1982-83, exposé no. 605, Astérisque 105-106 (1983), 149-166.

[Fack2] T. Fack, Sur la conjecture de Novikov, Index theory of elliptic operators, foliations, and operator algebras (J. Kaminker, K. C. Millett and C. Schochet, eds.), Contemp. Math., vol. 70, Amer. Math. Soc., Providence, RI, 1988, pp. 43-102.

[FarL] M. S. Farber and J. P. Levine, Jumps of the eta-invariant, with an appendix by S. Weinberger, preprint, Brandeis Univ., 1994.

[Far] F. T. Farrell, The obstruction to fibering a manifold over a circle, Indiana Univ. Math. J. 21 (1971), 315-346.

[FarHs1] F. T. Farrell and W. C. Hsiang, A geometric interpretation of the Künneth formula for algebraic K-theory, Bull. Amer. Math. Soc. 74 (1968), 548-553.

[FarHs2] F. T. Farrell and W.-C. Hsiang, A formula for $K_{1} R_{\alpha}[T]$, Applications of Categorical Algebra (New York, 1968), Proc. Symp. Pure Math., vol. 17, Amer. Math. Soc., Providence, 1970, pp. 192-218.

[FarHs3] F. T. Farrell and W.-C. Hsiang, Manifolds with $\pi_{1}=G \times_{\alpha} T$, Amer J. Math. 95 (1973), 813-848.

[FarHs4] F. T. Farrell and W.-C. Hsiang, Rational L-groups of Bieberbach groups, Comment. Math. Helv. 52 (1977), 89-109.

[FarHs5] F. T. Farrell and W.-C. Hsiang, On the rational homotopy groups of the diffeomorphism groups of discs, spheres and aspherical manifolds, Algebraic and geometric topology, Proc. Sympos. Pure Math., vol. 32 (Part 1), Amer. Math. Soc., Providence, RI, 1978, pp. 325-337.

[FarHs6] F. T. Farrell and W.-C. Hsiang, Remarks on Novikov's conjecture and the topological-Euclidean space form problem, Algebraic topology, Aarhus 1978, Lecture Notes in Math., vol. 763, Springer-Verlag, Berlin, Heidelberg, New York, 1979, pp. 635-642.

[FarHs7] F. T. Farrell and W.-C. Hsiang, The topological-Euclidean space form problem, Invent. Math. 45 (1978), 181-192.

[FarHs8] F. T. Farrell and W.-C. Hsiang, On Novikov's conjecture for nonpositively curved manifolds, I, Ann. of Math. 113 (1981), 199-209.

[FarHs9] F. T. Farrell and W.-C. Hsiang, The Whitehead group of poly-(finite or cyclic) groups, J. London Math. Soc. 24 (1981), 308-324.

[FarHs10] F. T. Farrell and W.-C. Hsiang, The stable topological-hyperbolic space form problems for complete manifolds of finite volume, Invent. Math. 69 (1982), $155-170$.

[FarHs11] F. T. Farrell and W.-C. Hsiang, Topological characterization of flat and almost flat Riemannian manifolds $M^{n}(n \neq 3,4)$, Amer. J. Math. 105 (1983), 641-672.

[FarHs12] F. T. Farrell and W.-C. Hsiang, On Novikov's conjecture for cocompact discrete subgroups of a Lie group, Algebraic topology, Aarhus 1982, Lecture Notes in Math., vol. 1051, Springer-Verlag, Berlin, Heidelberg, New York, 1984, pp. $38-48$. 
[FJ1] F. T. Farrell and L. E. Jones, Implications of the geometrization conjecture for the algebraic K-theory of 3-manifolds, Geometry and topology (Athens, GA, 1985), Lect. Notes in Pure and Appl. Math., vol. 105, Marcel Dekker, New York, 1987, pp. 109-113.

[FJ2] F. T. Farrell and L. E. Jones, $h$-cobordisms with foliated control, Bull. Amer. Math. Soc. 15 (1986), 69-72; Erratum, ibid. 16 (1987), 177.

[FJ3] F. T. Farrell and L. E. Jones, K-theory and dynamics, I, Ann. of Math. 124 (1986), 531-569; II, ibid. 126 (1987), 451-493.

[FJ4] F. T. Farrell and L. E. Jones, Topological rigidity for hyperbolic manifolds, Bull. Amer. Math. Soc. 19 (1988), 277-282.

[FJ5] F. T. Farrell and L. E. Jones, A topological analogue of Mostow's rigidity theorem, J. Amer. Math. Soc. 2 (1989), 257-370.

[FJ6] F. T. Farrell and L. E. Jones, Rigidity and other topological aspects of compact nonpositively curved manifolds, Bull. Amer. Math. Soc. 22 (1990), 59-64.

[FJ7] F. T. Farrell and L. E. Jones, Classical Aspherical Manifolds, Conf. Board of the Math. Sciences Regional Conf. Ser. in Math., vol. 75, Amer. Math. Soc., Providence, RI, 1990.

[FJ8] F. T. Farrell and L. E. Jones, Rigidity in geometry and topology, Proc. International Congress of Mathematicians (Kyoto, 1990), vol. 1, Math. Soc. Japan, Tokyo, 1991, pp. 653-663.

[FJ9] F. T. Farrell and L. E. Jones, Computations of stable pseudoisotopy spaces for aspherical manifolds, Algebraic Topology, Poznań 1989 (S. Jackowski, B. Oliver, and K. Pawałowski, eds.), Lecture Notes in Math., vol. 1474, SpringerVerlag, Berlin, Heidelberg, and New York, 1991, pp. 59-74.

[FJ10] F. T. Farrell and L. E. Jones, Stable pseudoisotopy spaces of compact non-positively curved manifolds, J. Diff. Geom. 34 (1991), 769-834.

[FJ11] F. T. Farrell and L. E. Jones, Foliated control without radius of injectivity restrictions, Topology 30 (1991), 117-142.

[FJ12] F. T. Farrell and L. E. Jones, Isomorphism conjectures in algebraic K-theory, J. Amer. Math. Soc. 6 (1993), 249-297.

[FJ13] F. T. Farrell and L. E. Jones, Topological rigidity for compact nonpositively curved manifolds, Differential Geometry (Los Angeles, 1990) (R. E. Greene and S.-T. Yau, eds.), Proc. Symp. Pure Math., vol. 54, Part 3, Amer. Math. Soc., Providence, 1993, pp. 229-274.

[Fer1] S. C. Ferry, Homotoping e-maps to homeomorphisms, Amer. J. Math 101 (1979), 567-582.

[Fer2] S. Ferry, Remarks on Steenrod homology, these Proceedings.

[FP] S. Ferry and E. K. Pedersen, Epsilon surgery theory, these Proceedings.

[FRR] S. Ferry, A. Ranicki, and J. Rosenberg, A history and survey of the Novikov Conjecture, these Proceedings.

[FRW] S. Ferry, J. Rosenberg, and S. Weinberger, Phénomènes de rigidité topologique équivariante, C. R. Acad. Sci. Paris Sér. I Math. 306 (1988), 777-782.

[FW1] S. Ferry and S. Weinberger, Curvature, tangentiality, and controlled topology, Invent. Math. 105 (1991), 401-414.

[FW2] S. Ferry and S. Weinberger, A coarse approach to the Novikov Conjecture, these Proceedings.

[FH] J. Fox and P. Haskell, K-theory and the spectrum of discrete subgroups of Spin(4, 1), Operator Algebras and Topology (Craiova, 1989), Pitman Res. Notes Math., vol. 270, Longman Sci. Tech., Harlow, 1992, pp. 30-44. 
[GM] I. M. Gelfand and A. S. Mishchenko, Quadratic forms over commutative group rings, and K-theory, Funkc. Anal i Priložen. 3 no. 4 (1969), 28-33; English translation, Funct. Anal. and its Appl. 3 (1969), 277-281.

[Gong] D. Gong, Equivariant Novikov Conjecture for groups acting on Euclidean buildings, preprint, Univ. of Chicago.

[GonL] D. Gong and K. Liu, On the rigidity of higher elliptic genera, preprint, Univ. of Chicago.

[Gor] V. V. Gorbatsevich, On some cohomology invariants of compact homogeneous manifolds, Lie groups, their discrete subgroups, and invariant theory, Adv. Soviet Math., vol. 8, Amer. Math. Soc., Providence, RI, 1992, pp. 69-85.

[Gr1] M. Gromov, Large Riemannian manifolds, Curvature and Topology of Riemannian Manifolds, Proc., Katata, 1985 (K. Shiohama, T. Sakai and T. Sunada, eds.), Lecture Notes in Math., vol. 1201, 1986, pp. 108-121.

[Gr2] M. Gromov, Hyperbolic groups, Essays in Group Theory (S. M. Gersten, ed.), Math. Sciences Research Inst. Publications, vol. 8, Springer-Verlag, New York, Berlin, Heidelberg, London, Paris, Tokyo, 1987, pp. 75-263.

[Gr3] M. Gromov, Kähler hyperbolicity and $L_{2}$-Hodge theory, J. Diff. Geometry 33 (1991), 263-292.

[Gr4] M. Gromov, Asymptotic invariants for infinite groups, Geometric Group Theory (G. A. Niblo and M. A. Roller, eds.), London Math. Soc. Lect. Notes, vol. 182, Cambridge Univ. Press, Cambridge, 1993, pp. 1-295.

[Gr5] M. Gromov, Geometric reflections on the Novikov Conjecture, these Proceedings.

[Gr6] M. Gromov, Positive curvature, macroscopic dimension, spectral gaps and higher signatures, Functional Analysis on the Eve of the 21st Century (Proc. conf. in honor of I. M. Gelfand's 80th birthday) (S. Gindikin, J. Lepowsky and R. Wilson, eds.), Progress in Math., Birkhäuser, Boston, 1995 (to appear).

[GL1] M. Gromov and H. B. Lawson, Jr., Spin and scalar curvature in the presence of a fundamental group. I, Ann. of Math. 111 (1980), 209-230.

[GL2] M. Gromov and H. B. Lawson, Jr., Positive scalar curvature and the Dirac operator on complete Riemannian manifolds, Publ. Math. Inst. Hautes Études Sci. 58 (1983), 83-196.

[HaMTW] I. Hambleton, J. Milgram, L. Taylor and B. Williams, Surgery with finite fundamental group, Proc. London Math. Soc. 56 (1988), 349-379.

[Has] P. Haskell, Direct limits in an equivariant $K$-theory defined by proper cocycles, Michigan Math. J. 36 (1989), 17-27.

[Hi] G. Higman, Units in group rings, Proc. Lond. Math. Soc. (2) 46 (1940), $231-248$.

[Hig1] N. Higson, A primer on $K K$-theory, Operator theory: operator algebras and applications (Durham, NH, 1988), Proc. Sympos. Pure Math., vol. 51, Part 1, Amer. Math. Soc., Providence, RI, 1990, pp. 239-283.

[Hig2] N. Higson, On the relative K-homology theory of Baum and Douglas, Unpublished preprint, 1990.

[Hig3] N. Higson, On the cobordism invariance of the index, Topology 30 (1991), 439-443.

[HigR1] N. Higson and J. Roe, A homotopy invariance theorem in coarse cohomology and K-theory, Trans. Amer. Math. Soc. 345 (1994), 347-365.

[HigR2] N. Higson and J. Roe, On the coarse Baum-Connes conjecture, these Proceedings. 
[HigRY] N. Higson, J. Roe, and G. Yu, A coarse Mayer-Vietoris principle, Math. Proc. Camb. Philos. Soc. 114 (1993), 85-97.

[Hil] M. Hilsum, Fonctorialité en K-théorie bivariante pour les variétés lipschitziennes, K-Theory 3 (1989), 401-440.

[HilS] M. Hilsum and G. Skandalis, Invariance par homotopie de la signature à coefficients dans un fibré presque plat, J. reine angew. Math. 423 (1992), 73-99.

[Hir] F. Hirzebruch, The signature theorem: reminiscences and recreation, Prospects in mathematics (Proc. Sympos., Princeton Univ., Princeton, N.J., 1970), Ann. of Math. Studies, vol. 70, Princeton Univ. Press, Princeton, NJ, 1971, pp. 3-31.

[Ho] J. G. Hollingsworth, A short list of problems, Geometric topology (Proc. Georgia Topology Conf., Athens, Ga., 1977), Academic Press, New York and London, 1979, pp. 685-692.

[Hs1] W.-C. Hsiang, On $\pi_{i}$ Diff $M^{n}$, Geometric topology (Proc. Georgia Topology Conf., Athens, Ga., 1977), Academic Press, New York and London, 1979, pp. 351-365.

[Hs2] W.-C. Hsiang, Geometric applications of algebraic K-theory, Proc. International Congress of Mathematicians (Warsaw, 1983), Polish Scientific Publishers, Warsaw, 1984, pp. 99-119.

[Hs3] W.-C. Hsiang, Borel's conjecture, Novikov's conjecture and the K-theoretic analogues, Algebra, analysis and geometry (Taipei, 1988), World Sci. Publishing, Teaneck, NJ, 1989, pp. 39-58.

[HsR] W.-C. Hsiang and H. D. Rees, Mishchenko's work on Novikov's conjecture, Operator algebras and K-theory (San Francisco, 1981) (R. G. Douglas and C. Schochet, eds.), Contemp. Math., vol. 10, Amer. Math. Soc., Providence, RI, 1982, pp. 77-98.

[HsS] W.-C. Hsiang and J. Shaneson, Fake tori, the annulus conjecture, and the conjectures of Kirby, Proc. Nat. Acad. Sci. U.S.A. 62 (1969), 687-691.

$[\mathrm{Hu}] \quad$ B. $\mathrm{Hu}$, Whitehead groups of finite polyhedra with nonpositive curvature, J. Diff. Geom. 38 (1993), 501-517.

[Hu1] S. Hurder, Eta invariants and the odd index theorem for coverings, Geometric and topological invariants of elliptic operators (Bowdoin Coll., 1988) (J. Kaminker, ed.), Contemp. Math., vol. 105, Amer. Math. Soc., Providence, RI, 1990, pp. 47-82.

[Hu2] S. Hurder, Topology of covers and spectral theory of geometric operators, Index Theory and Operator Algebras (Boulder, CO, 1991) (J. Fox and P. Haskell, eds.), Contemp. Math., vol. 148, Amer. Math. Soc., Providence, RI, 1993, pp. 87-119.

[Hu3] S. Hurder, Exotic index theory and the Novikov Conjecture, these Proceedings.

[Hu4] S. Hurder, Exotic index theory for foliations, preprint, Univ. of Illinois at Chicago.

[Ji1] R. Ji, Smooth dense subalgebras of reduced group $C^{*}$-algebras, Schwartz cohomology of groups, and cyclic cohomology, J. Functional Analysis 107 (1992), $1-33$.

[Ji2] R. Ji, Some applications of cyclic cohomology to the study of group $C^{*}$-algebras, Index Theory and Operator Algebras (Boulder, CO, 1991) (J. Fox and P. Haskell, eds.), Contemp. Math., vol. 148, Amer. Math. Soc., Providence, RI, 1993, pp. 121-129.

[Ji3] R. Ji, Nilpotency of Connes' periodicity operator and the idempotent conjectures, $K$-Theory (to appear). 
[Jo1] P. Jolissaint, $K$-theory of reduced $C^{*}$-algebras and rapidly decreasing functions on groups, $K$-Theory 2 (1989), 723-736.

[Jo2] P. Jolissaint, Rapidly decreasing functions in reduced $C^{*}$-algebras of groups, Trans. Amer. Math. Soc. 317 (1990), 167-196.

[Ju1] P. Julg, $C^{*}$-algèbres associées à des complexes simpliciaux, C. R. Acad. Sci. Paris Sér. I Math. 308 (1989), 97-100.

[Ju2] P. Julg, $K$-théorie des $C^{*}$-algèbres associées à certains groupes hyperboliques, Dissertation, Univ. Louis Pasteur, Publ. Inst. Recherche Math. Avancée, Univ. Louis Pasteur, Strasbourg, 1991.

[JuK] P. Julg and G. Kasparov, L'anneau $K K_{G}(\mathbb{C}, \mathbb{C})$ pour $G=S U(n, 1)$, C. R. Acad. Sci. Paris Sér. I Math. 313 (1991), 259-264.

[JuV] P. Julg and A. Valette, Fredholm modules associated to Bruhat-Tits buildings, Proc. Miniconferences on Harmonic Analysis and Operator Algebras (Canberra, 1987) (M. Cowling, C. Meaney, and W. Moran, eds.), Proc. Centre for Mathematical Analysis, vol. 16, Centre for Mathematical Analysis, Australian National Univ., Canberra, 1987, pp. 143-155.

[KM1] J. Kaminker and J. Miller, A comment on the Novikov conjecture, Proc. Amer. Math. Soc. 83 (1981), 656-658.

[KM2] J. Kaminker and J. Miller, Homotopy invariance of the analytic index of signature operators over $C^{*}$-algebras, J. Operator Theory 14 (1985), 113-127.

[Kas1] G. G. Kasparov, A generalized index for elliptic operators, Funkc. Anal i Priložen. 7 no. 3 (1973), 82-83; English translation, Funct. Anal. Appl. 7 (1973), 238-240.

[Kas2] G. G. Kasparov, Topological invariants of elliptic operators, I: K-homology, Izv. Akad. Nauk SSSR, Ser. Mat. 39 (1975), 796-838; English translation, Math. USSR-Izv. 9 (1975), 751-792.

[Kas3] G. G. Kasparov, The operator $K$-functor and extensions of $C^{*}$-algebras, Izv. Akad. Nauk SSSR, Ser. Mat. 44 (1980), 571-636; English translation, Math. USSR-Izv. 16 (1981), 513-572.

[Kas4] G. G. Kasparov, K-theory, group $C^{*}$-algebras, and higher signatures: Conspectus, I, II, preprint, Inst. for Chemical Physics of the Soviet Acad. of Sci., Chernogolovka; reprinted with annotations, these Proceedings.

[Kas5] G. G. Kasparov, Operator K-theory and its applications: elliptic operators, group representations, higher signatures, $C^{*}$-extensions, Proc. International Congress of Mathematicians (Warsaw, 1983), vol. 2, Polish Scientific Publishers, Warsaw, 1984, pp. 987-1000.

[Kas6] G. G. Kasparov, Operator K-theory and its applications, Current problems in mathematics. Newest results, Itogi Nauki i Tekhniki, vol. 27, Akad. Nauk SSSR, Vsesoyuz. Inst. Nauchn. i Tekhn. Inform., Moscow, 1985, pp. 3-31.

[Kas7] _ Equivariant KK-theory and the Novikov conjecture, Invent. Math. 91 (1988), 147-201.

[Kas8] - Novikov's conjecture on higher signatures: the operator K-theory approach, Representation Theory of Groups and Algebras (J. Adams, R. Herb, S. Kudla, J.-S. Li, R. Lipsman, and J. Rosenberg, eds.), Contemporary Math., vol. 145, Amer. Math. Soc., Providence, 1993, pp. 79-100.

[KS1] G. G. Kasparov and G. Skandalis, Groupes agissant sur des immeubles de Bruhat-Tits, K-théorie operatorielle et conjecture de Novikov, C. R. Acad. Sci. Paris Sér. I Math. 310 (1990), 171-174.

[KS2] G. G. Kasparov and G. Skandalis, Groups acting on buildings, operator K-theo- 
ry, and Novikov's conjecture, K-Theory 4, Special issue in honor of A. Grothendieck (1991), 303-337.

[KS3] G. G. Kasparov and G. Skandalis, Groupes "boliques" et conjecture de Novikov, C. R. Acad. Sci. Paris Sér. I Math. 319 (1994), 815-820.

[Kee] J. Keesling, The one-dimensional Čech cohomology of the Higson corona and its compactification, preprint, Univ. of Florida (1994).

[KerM] M. Kervaire and J. Milnor, Groups of homotopy spheres, I, Ann. of Math. 77 (1963), 504-537.

[KirS] R. Kirby and L. Siebenmann, Foundational Essays on Topological Manifolds, Smoothings, and Triangulations, Ann. of Math. Studies, vol. 88, Princeton Univ. Press, Princeton, NJ, 1977.

[Kis] J. Kister, Microbundles are fiber bundles, Ann. of Math. 80 (1964), 190-199.

[LaMi] H. B. Lawson, Jr. and M.-L. Michelsohn, Spin Geometry, Princeton Math. Ser., vol. 38, Princeton Univ. Press, Princeton, NJ, 1990.

[LeRa] N. Levitt and A. A. Ranicki, Intrinsic transversality structures, Pacific J. Math. 129 (1987), 85-144.

[Lod] J.-L. Loday, K-théorie algébrique et représentations des groupes, Ann. Sci. École Norm. Sup. (4) 9 (1976), 309-377.

[LodQ] J.-L. Loday and D. Quillen, Cyclic homology and the Lie algebra homology of matrices, Comment. Math. Helv. 59 (1984), 565-591.

[Lott] J. Lott, Superconnections and higher index theory, Geom. Funct. Anal. 4 (1992), 421-454.

[Lott] J. Lott, Higher eta-invariants, K-Theory 6 (1992), 191-233.

[Lus] G. Lusztig, Novikov's higher signature and families of elliptic operators, J. Diff. Geom. 7 (1972), 229-256.

[Mac] M. Macho-Stadler, La conjecture de Baum-Connes pour un feuilletage sans holonomie de codimension un sur une variété fermée, Publ. Mat. 33 (1989), 445-457.

[Ma] I. Madsen, Reidemeister torsion, surgery invariants and spherical space forms, Proc. London Math. Soc. 46 (1983), 193-240.

[Mat1] V. Mathai, On the homotopy invariance of reduced eta and other signature type invariants, preprint, Univ. of Adelaide.

[Mat2] V. Mathai, Spectral flow, eta invariants, and von Neumann algebras, J. Funct. Anal. 109 (1992), 442-456.

[Milg] R. J. Milgram, Surgery with finite fundamental group, II: the oozing conjecture, Pacific J. Math. 151 (1991), 117-150.

[MilgR] R. J. Milgram and A. A. Ranicki, The L-theory of Laurent polynomial extensions and genus 0 function fields, J. reine angew. Math. 406 (1990), 121-166.

[Mill] J. Miller, Signature operators and surgery groups over $C^{*}$-algebras, Preprint, Indiana Univ./Purdue Univ. (1992).

[Miln1] J. Milnor, On manifolds homeomorphic to the 7-sphere, Ann. of Math. 64 (1956), 399-405.

[Miln2] J. Milnor, A procedure for killing the homotopy groups of differentiable manifolds, Differential Geometry (Tucson, 1960) (C. B. Allendoerfer, ed.), Proc. Symp. Pure Math., vol. 3, 1961, pp. 39-55.

[Mis1] A. S. Mishchenko, Homotopy invariants of nonsimply connected manifolds, I: rational invariants, Izv. Akad. Nauk SSSR Ser. Mat. 34 (1970), 501-514; English translation, Math. USSR-Izv. 4 (1970), 506-519.

[Mis2] A. S. Mishchenko, Homotopy invariants of nonsimply connected manifolds, III: 
Higher signatures, Izv. Akad. Nauk SSSR Ser. Mat. 35 (1971), 1316-1355; Erratum , ibid. 36 (1972), 910.

[Mis3] A. S. Mishchenko, Infinite dimensional representations of discrete groups, and higher signatures, Izv. Akad. Nauk SSSR Ser. Mat. 38 (1974), 81-106; English translation, Math. USSR-Izv. 8 (1974), 85-111.

[Mis4] A. S. Mishchenko, On Fredholm representations of discrete groups, Funkc. Anal i Priložen. 9 no. 2 (1975), 36-41; English translation, Funct. Anal. Appl. 9 (1975), 121-125.

[Mis5] A. S. Mishchenko, Hermitian K-theory. The theory of characteristic classes and methods of functional analysis, Uspekhi Mat. Nauk 31 no. 2 (188) (1976), 69-134; English translation, Russian Math. Surv. 31 no. 2 (1972), 71-138.

[Mis6] A. S. Mishchenko, $C^{*}$-algebras and K-theory, Algebraic Topology, Århus 1978 (J. L. Dupont and I. Madsen, eds.), Lecture Notes in Math., vol. 763, SpringerVerlag, Berlin, Heidelberg, and New York, 1979, pp. 262-274.

[MisF] A. S. Mishchenko and A. T. Fomenko, The index of elliptic operators over $C^{*}$-algebras, Izv. Akad. Nauk SSSR Ser. Mat. 43 (1979), 831-859; English translation, Math. USSR-Izv. 15 (1980), 87-112.

[MisS1] A. S. Mishchenko and Y. P. Soloviev, A classifying space for hermitian K-theory, Trudy Sem. Vect. and Tensor Anal. 18 (1976), 140-168.

[MisS2] A. S. Mishchenko and Yu. P. Soloviev, Infinite-dimensional representations of fundamental groups, and formulas of Hirzebruch type, Dokl. Akad. Nauk SSSR 234 (1977), 761-764; English translation, Soviet Math. Dokl. 18 (1977), $767-771$.

[MisS3] A. S. Mishchenko and Yu. P. Soloviev, Representations of Banach algebras and formulas of Hirzebruch type, Mat. Sb. 111(153) (1980), 209-226; English translation, Math. USSR-Sb. 39 (1980), 189-205.

[Mos] H. Moscovici, Cyclic cohomology and invariants of multiply connected manifolds, Proc. International Congress of Mathematicians (Kyoto, 1990), vol. 1, Math. Soc. Japan, Tokyo, 1991, pp. 675-688.

[Na1] T. Natsume, The Baum-Connes conjecture, the commutator theorem, and Rieffel projections, C. R.-Math. Rep. Acad. Sci. Canada 10 (1988), 13-18.

[Na2] T. Natsume, Topological $K$-theory for codimension one foliations without holonomy, Foliations (Tokyo, 1983), Adv. Stud. Pure Math., vol. 5, 1985, pp. 15-27.

[Ne] W. D. Neumann, Signature-related invariants of manifolds, I: monodromy and $\gamma$-invariants, Topology 18 (1979), 147-160.

[Ni1] A. J. Nicas, Induction theorems for groups of homotopy manifold structures, Mem. Amer. Math. Soc. 39 no. 267 (1982).

[Ni2] A. J. Nicas, On $\mathrm{Wh}_{3}$ of a Bieberbach group, Math. Proc. Cambridge Philos. Soc. 95 (1984), 55-60.

[Ni3] A. J. Nicas, On the higher Whitehead groups of a Bieberbach group, Trans. Amer. Math. Soc. 287 (1985), 853-859.

[Nis1] V. Nistor, Group cohomology and the cyclic cohomology of crossed products, Invent. Math. 99 (1990), 411-424.

[Nis2] V. Nistor, Cyclic cohomology of crossed products by algebraic groups, Invent. Math. 112 (1993), 615-638.

[Nov1] S. P. Novikov, Diffeomorphisms of simply connected manifolds, Dokl. Akad. Nauk SSSR 143 (1962), 1046-1049; English translation, Soviet Math. Dokl. 3 (1962), 540-543.

[Nov2] S. P. Novikov, Homotopically equivalent smooth manifolds. I, Izv. Akad. Nauk 
SSSR, Ser. Mat. 28 (1964), 365-474; English translation, Amer. Math. Soc. Transl. (2) 48 (1965), 271-396.

[Nov3] S. P. Novikov, Rational Pontrjagin classes. Homeomorphism and homotopy type of closed manifolds. I, Izv. Akad. Nauk SSSR, Ser. Mat. 29 (1965), 1373-1388.

[Nov4] S. P. Novikov, The homotopy and topological invariance of certain rational Pontrjagin classes, Dokl. Akad. Nauk SSSR 162 (1965), 1248-1251; English translation, Soviet Math. Dokl. 6 (1965), 854-857.

[Nov5] S. P. Novikov, Topological invariance of rational classes of Pontrjagin, Dokl. Akad. Nauk SSSR 163 (1965), 298-300; English translation, Soviet Math. Dokl. 6 (1965), 921-923.

[Nov6] S. P. Novikov, On manifolds with free abelian fundamental group and their application, Izv. Akad. Nauk SSSR, Ser. Mat. 30 (1966), 207-246; English translation, Amer. Math. Soc. Transl. (2) 71 (1968), 1-42.

[Nov7] S. P. Novikov, Pontrjagin classes, the fundamental group and some problems of stable algebra, Proc. International Congress of Mathematicians (Moscow, 1966), Amer. Math. Soc. Transl. (2), vol. 70, Amer. Math. Soc., Providence, RI, 1968, pp. 172-179.

[Nov8] S. P. Novikov, Analogues hermitiens de la K-théorie, Actes du Congrès International des Mathématiciens (Nice, 1970), vol. 2, Gauthier-Villars, Paris, pp. 39-45.

[Nov9] S. P. Novikov, Pontrjagin classes, the fundamental group and some problems of stable algebra, Essays on Topology and Related Topics (Mémoires dédiés à Georges de Rham) (A. Haefliger and R. Narasimhan, eds.), Springer-Verlag, New York, Heidelberg, and Berlin, 1970, pp. 147-155.

[Nov10] S. P. Novikov, Algebraic construction and properties of Hermitian analogues of $K$-theory over rings with involution from the viewpoint of the Hamiltonian formalism. Applications to differential topology and the theory of characteristic classes, Izv. Akad. Nauk SSSR, Ser. Mat. 34 (1970), 253-288, 475-500; English translation, Math. USSR-Izv. 4 (1970), 257-292, 479-505.

[Ogle1] C. Ogle, Assembly maps, K-theory, and hyperbolic groups, K-Theory 6 (1992), 235-265.

[Ogle2] C. Ogle, Simplicial rapid decay algebras associated to a discrete group, Preprint, Ohio State Univ., 1995.

[Ogle3] C. Ogle, Filtrations of simplicial functors, topological K-theory and the strong Novikov conjecture, Preprint, Ohio State Univ., 1995.

[Pe1] E. K. Pedersen, On the bounded and thin h-cobordism theorem parametrized by $\mathbb{R}^{k}$, Transformation Groups, Poznań 1985 (S. Jackowski and K. Pawałowski, eds.), Lecture Notes in Math., vol. 1217, Springer-Verlag, Berlin, Heidelberg, and New York, 1986, pp. 306-320.

[Pe2] E. K. Pedersen, Bounded and continuous control, these Proceedings.

[PeRW] E. K. Pedersen, J. Roe and S. Weinberger, On the homotopy invariance of the boundedly controlled analytic signature of a manifold over an open cone, these Proceedings.

[PeW] E. K. Pedersen and C. Weibel, K-theory homology of spaces, Algebraic Topology, Proc., 1986 (G. Carlsson R. L. Cohen, H. R. Miller, and D. C. Ravenel, eds.), Lecture Notes in Math., vol. 1370, Springer-Verlag, Berlin, Heidelberg, and New York, 1989, pp. 346-361.

[Pet] M. E. Petty, Waldhausen's theory of $k$-fold end structures, Topology Appl. 14 
(1982), 71-85.

[Pim1] M. Pimsner, KK-groups of crossed products by groups acting on trees, Invent. Math. 86 (1986), 603-634.

[Pim2] M. Pimsner, K-theory for groups acting on trees, Proc. International Congress of Mathematicians (Kyoto, 1990), vol. 1, Math. Soc. Japan, Tokyo, 1991, pp. 979-986.

[PimV1] M. Pimsner and D. Voiculescu, Exact sequences for K-groups and Ext-groups for certain cross products of $C^{*}$-algebras, J. Operator Theory 4 (1980), 93-118.

[PimV2] M. Pimsner and D. Voiculescu, K-groups of reduced crossed products by free groups, J. Operator Theory 8 (1982), 131-156.

[Pl1] R. Plymen, K-theory for the reduced $C^{*}$-algebra of $S L_{2}\left(\mathbb{Q}_{p}\right)$, Operator Algebras and their Connections with Topology and Ergodic Theory (H. Araki, C. C. Moore, Ş. Strătilă and D. Voiculescu, eds.), Lect. Notes in Math., vol. 1132, Springer-Verlag, Berlin, Heidelberg, New York, 1985, pp. 409-420.

[Pl2] R. Plymen, Reduced $C^{*}$-algebra of the $p$-adic group $G L(n)$, J. Funct. Anal. 72 (1987), 1-12.

[Pl3] R. Plymen, Reduced $C^{*}$-algebra for reductive $p$-adic groups, J. Funct. Anal. 88 (1990), 251-266.

[Q1] F. Quinn, A geometric formulation of surgery, Topology of manifolds (Athens, Ga. 1969) (J. C. Cantrell and C. H. Edwards, ed.), Markham Math. Ser., Markham Press, Chicago, 1970, pp. 500-511.

[Q2] F. Quinn, $B_{\left(T O P_{n}\right)} \sim$ and the surgery obstruction, Bull. Amer. Math. Soc. 77 (1971), 596-600.

[Q3] F. Quinn, Ends of maps, II, Invent. Math. 68 (1982), 353-424.

[Q4] F. Quinn, Algebraic K-theory of poly-(finite or cyclic) groups, Bull. Amer. Math. Soc. 12 (1985), 221-226.

[Q5] F. Quinn, Applications of topology with control, Proc. International Congress of Mathematicians (Berkeley, Calif., 1986), vol. 1, Amer. Math. Soc., Providence, RI, 1987, pp. 598-606.

[Q6] F. Quinn, Homotopically stratified spaces, J. Amer. Math. Soc. 1 (1988), 441499.

[Q7] F. Quinn, Assembly maps in bordism-type theories, these Proceedings.

[Ran1] A. Ranicki, Algebraic L-theory I. Foundations, Proc. London Math. Soc. 27 (1973), 101-125.

[Ran2] A. Ranicki, Algebraic L-theory II. Laurent extensions, Proc. London Math. Soc. 27 (1973), 126-158.

[Ran3] A. Ranicki, Algebraic L-theory III. Twisted Laurent extensions, Hermitian $K$ Theory and Geometric Applications (H. Bass, ed.), Lecture Notes in Math., vol. 343, Springer-Verlag, Berlin, Heidelberg, and New York, 1973, pp. 412-463.

[Ran4] A. Ranicki, The algebraic theory of surgery I. Foundations, Proc. London Math. Soc. 40 (1980), 87-192.

[Ran5] A. Ranicki, The algebraic theory of surgery II. Applications to topology, Proc. London Math. Soc. 40 (1980), 193-283.

[Ran6] A. Ranicki, The total surgery obstruction, Algebraic topology, Aarhus 1978, Lecture Notes in Math., vol. 763, Springer-Verlag, Berlin, Heidelberg, New York, 1979, pp. 275-316.

[Ran7] A. Ranicki, Exact Sequences in the Algebraic Theory of Surgery, Princeton Math. Notes, vol. 26, Princeton Univ. Press, Princeton, 1981.

[Ran8] A. Ranicki, Lower K- and L-Theory, London Math. Soc. Lecture Notes, vol. 178, 
Cambridge Univ. Press, Cambridge, 1992.

[Ran9] A. Ranicki, Algebraic L-Theory and Topological Manifolds, Cambridge Tracts in Math., vol. 102, Cambridge Univ. Press, Cambridge, 1992.

[Ran10] A. Ranicki, On the Novikov conjecture, these Proceedings.

[Ran11] A. Ranicki (editor), The Hauptvermutung Book, papers by A. J. Casson, D. P. Sullivan, M. A. Armstrong, G. E. Cooke, C. P. Rourke and A. A. Ranicki, $K$-Theory book series, vol. 1 (to appear).

[Roe1] J. Roe, An index theorem on open manifolds, I, J. Diff. Geom. 27 (1988), 87-113; II, ibid., 115-136.

[Roe2] J. Roe, Hyperbolic metric spaces and the exotic cohomology Novikov conjecture, K-Theory 4 (1990), 501-512; Erratum, ibid. 5 (1991), 189.

[Roe3] J. Roe, Coarse cohomology and index theory on complete Riemannian manifolds, Mem. Amer. Math. Soc. no. 497 (1993).

[Rokh] V. A. Rokhlin, Pontrjagin-Hirzebruch class of codimension 2, Izv. Akad. Nauk SSSR Ser. Mat. 30 (1966), 705-718.

[RokS] V. A. Rokhlin and A. S. Shvarts, The combinatorial invariance of Pontrjagin classes, Dokl. Akad. Nauk SSSR 114 (1957), 490-493.

[Ros1] J. Rosenberg, Group $C^{*}$-algebras and topological invariants, Operator algebras and group representations, Vol. II (Neptun, 1980), Monographs Stud. Math., vol. 18, Pitman, Boston and London, 1984, pp. 95-115.

[Ros2] J. Rosenberg, $C^{*}$-algebras, positive scalar curvature, and the Novikov conjecture, Publ. Math. Inst. Hautes Études Sci. 58 (1983), 197-212.

[Ros3] J. Rosenberg, $C^{*}$-algebras, positive scalar curvature, and the Novikov conjecture, II, Geometric methods in operator algebras (Kyoto, 1983) (H. Araki and E. G. Effros, eds.), Pitman Res. Notes Math., vol. 123, Longman Sci. Tech., Harlow, 1986, pp. 341-374.

[Ros4] J. Rosenberg, $C^{*}$-algebras, positive scalar curvature, and the Novikov conjecture, III, Topology 25 (1986), 319-3362.

[Ros5] J. Rosenberg, The KO-assembly map and positive scalar curvature, Algebraic Topology, Poznań 1989 (S. Jackowski, B. Oliver, and K. Pawałowski, eds.), Lecture Notes in Math., vol. 1474, Springer-Verlag, Berlin, Heidelberg, and New York, 1991, pp. 170-182.

[Ros6] J. Rosenberg, Analytic Novikov for topologists, these Proceedings.

[RS1] J. Rosenberg and S. Stolz, Manifolds of positive scalar curvature, Algebraic Topology and its Applications (G. Carlsson, R. Cohen, W.-C. Hsiang, and J. D. S. Jones, eds.), M. S. R. I. Publications, vol. 27, Springer-Verlag, New York, 1994, pp. 241-267.

[RS2] J. Rosenberg and S. Stolz, A "stable" version of the Gromov-Lawson conjecture, The Čech Centennial: Proc. Conf. on Homotopy Theory (M. Cenkl and H. Miller, eds.), Contemp. Math., vol. 181, Amer. Math. Soc., Providence, RI, 1995, pp. 405-418.

[RS3] J. Rosenberg and S. Stolz, Metrics of Positive Scalar Curvature, DMV-Seminar, Birkhäuser, Basel, Boston, in preparation.

[RW1] J. Rosenberg and S. Weinberger, Higher G-indices and applications, Ann. Sci. École Norm. Sup. 21 (1988), 479-495.

[RW2] J. Rosenberg and S. Weinberger, An equivariant Novikov conjecture, with an appendix by J. P. May, $K$-Theory 4, Special issue in honor of A. Grothendieck (1990), 29-53.

[RW3] J. Rosenberg and S. Weinberger, Higher G-signatures for Lipschitz manifolds, 
K-Theory 7 (1993), 101-132.

[Sh1] J. Shaneson, Wall's surgery obstruction groups for $\mathbb{Z} \times G$, for suitable groups G, Bull. Amer. Math. Soc. 74 (1968), 467-471.

[Sh2] J. Shaneson, Wall's surgery obstruction groups for $\mathbb{Z} \times G$, Ann. of Math. 90 (1969), 296-334.

[Shar] B. L. Sharma, Topologically invariant characteristic classes, Topology Appl. 21 (1985), 135-146.

[SharSi] B. L. Sharma and N. Singh, Topological invariance of integral Pontrjagin classes mod p, Topology Appl. 63 (1995), 59-67.

[Si] N. Singh, On topological and homotopy invariance of integral Pontrjagin classes modulo a prime p, Topology Appl. 38 (1991), 225-235.

[Sk1] G. Skandalis, Kasparov's bivariant K-theory and applications, Expositiones Math. 9 (1991), 193-250.

[Sk2] G. Skandalis, Approche de la conjecture de Novikov par la cohomologie cyclique (d'après A. Connes, M. Gromov et H. Moscovici), Séminaire Bourbaki, 1990-91, exposé no. 739, Astérisque 201-203 (1992), 299-320.

[So] Yu. P. Soloviev, A theorem of Atiyah-Hirzebruch type for infinite groups, Vestnik Moskov. Univ., Ser. I-Mat. Meh. 30 no. 4 (1975), 26-35; English translation, Moscow Univ. Math. Bull. 30 no. 3/4 (1975), 77-85.

[Spv] M. Spivak, Spaces satisfying Poincaré duality, Topology 6 (1967), 77-102.

[Sul] D. Sullivan, Triangulating homotopy equivalences, Ph.D. dissertation, Princeton Univ., Princeton, 1965.

[Tak1] H. Takai, A counterexample of strong Baum-Connes conjectures for foliated manifolds, Mappings of operator algebras (Philadelphia, PA, 1988), Progress in Math., vol. 84, Birkhäuser, Boston, 1990, pp. 183-197.

[Tak2] H. Takai, Baum-Connes conjectures and their applications, Dynamical systems and applications (Kyoto, 1987), World Sci. Adv. Ser. Dyn. Syst., vol. 5, World Sci., Singapore, 1987, pp. 89-116.

[Tak3] H. Takai, On the Baum-Connes conjecture, The study of dynamical systems (Kyoto, 1989), World Sci. Adv. Ser. Dyn. Syst., vol. 7, World Sci., Teaneck, NJ, 1989, pp. 149-154.

[TaW] L. Taylor and B. Williams, Surgery spaces: formulae and structure, Algebraic topology, Waterloo, 1978, Lecture Notes in Math., vol. 741, Springer-Verlag, Berlin, Heidelberg, New York, 1979, pp. 170-195.

[Th1] R. Thom, Quelques propriétés globales des variétés différentiables, Comment. Math. Helv. 28 (1954), 17-86.

[Th2] R. Thom, Les classes caractéristiques de Pontrjagin des variétés triangulées, Proc. Symp. Internacional de Topologica Algebraica, Univ. Nacional Autonoma de México, Mexico City, 1958, pp. 54-67.

[Ths1] C. B. Thomas, Splitting theorems for certain PD ${ }^{3}$-groups, Math. Z. 186 (1984), 201-209.

[Ths2] C. B. Thomas, 3-manifolds and PD(3)-groups, these Proceedings.

[Ti] U. Tillmann, K-theory of fine topological algebras, Chern character, and assembly, K-Theory 6 (1992), 57-86.

[To] A. M. Torpe, K-theory for the leaf space of foliations by Reeb components, J. Funct. Anal. 61 (1985), 15-71.

[Tr1] E. V. Troitsky, The index of the generalized Hirzebruch operator and the homotopically invariant higher signatures, Selected topics in Algebra, Geometry and Discr. Math., Moscow State Univ., Moscow, 1992, pp. 151-152. 
[Tr2] E. V. Troitsky, The Hirzebruch operator on PL manifolds and the higher signatures, Uspekhi Mat. Nauk 48 no. 1 (1993), 189-190; English translation, Russian Math. Surv. 48 no. 1 (1993), 197-198.

[Tsy] B. L. Tsygan, Homology of matrix Lie algebras over rings and Hochschild homology, Uspekhi Mat. Nauk 38 no. 2 (1983), 217-218; English translation, Russian Math. Surv. 38 no. 2 (1983), 198-199.

[Va] A. Valette, Le rôle des fibrés de rang fini en théorie de Kasparov équivariante, Mem. Acad. Roy. Belg., Classes des Sciences (1986).

[Vi] M. F. Vigneras, Homologie cyclique, principe de Selberg et pseudo-coefficient, Invent. Math. 116 (1994), 651-676.

[Wald1] F. Waldhausen, Algebraic K-theory of amalgamated free products, Ann. of Math. 108 (1978), 135-256.

[Wald2] F. Waldhausen, Algebraic K-theory of topological spaces, I, Algebraic and Geometric Topology (Stanford, 1976), Proc. Symp. Pure Math., vol. 32, Amer. Math. Soc., Providence, RI, 1978, pp. 35-60; II, Algebraic topology, Aarhus 1978, Lecture Notes in Math., vol. 763, Springer-Verlag, Berlin, Heidelberg, New York, 1979, pp. 356-394.

[Wald3] F. Waldhausen, Algebraic K-theory of spaces, a manifold approach, Current Trends in Algebraic Topology, Part 1 (London, Ont., 1981), Canad. Math. Soc. Conf. Proc., vol. 2, Amer. Math. Soc., Providence, RI, 1982, pp. 141-184.

[Wall1] C. T. C. Wall, Surgery of non-simply-connected manifolds, Ann. of Math. 84 (1966), 217-276.

[Wall2] C. T. C. Wall, Surgery on Compact Manifolds, London Math. Soc. Monographs, vol. 1, Academic Press, London and New York, 1970.

[Was] A. Wassermann, A proof of the Connes-Kasparov conjecture for connected reductive linear Lie groups, C. R. Acad. Sci. Paris Sér. I Math. 304 (1987), 559-562.

[W1] S. Weinberger, The Novikov conjecture and low-dimensional topology, Comment. Math. Helv. 58 (1983), 355-364.

[W2] S. Weinberger, Constructions of group actions: a survey of some recent developments, Group actions on manifolds (Boulder, Colo., 1983) (R. Schultz, ed.), Contemp. Math., vol. 36, Amer. Math. Soc., Providence, RI, 1985, pp. 269-298.

[W3] S. Weinberger, Group actions and higher signatures, Proc. Nat. Acad. Sci. U.S.A. 82 (1985), 1297-1298.

[W4] S. Weinberger, Homologically trivial group actions, II: nonsimply connected manifolds, Amer. J. Math. 108 (1986), 1259-1275.

[W5] S. Weinberger, Group actions and higher signatures, II, Comm. Pure Appl. Math. 40 (1987), 179-187.

[W6] S. Weinberger, Class numbers, the Novikov Conjecture, and transformation groups, Topology 27 (1988), 353-365.

[W7] S. Weinberger, Homotopy invariance of eta invariants, Proc. Nat. Acad. Sci. U.S.A. 85 (1988), 5362-5363.

[W8] S. Weinberger, Aspects of the Novikov conjecture, Geometric and topological invariants of elliptic operators (Bowdoin Coll., 1988) (J. Kaminker, ed.), Contemp. Math., vol. 105, Amer. Math. Soc., Providence, RI, 1990, pp. 281-297.

[W9] S. Weinberger, The Topological Classification of Stratified Spaces, Chicago Lectures in Math., Univ. of Chicago Press, Chicago, 1994.

[W10] S. Weinberger, Nonlocally linear manifolds and orbifolds, Proc. International Congress of Mathematicians (Zürich, 1994) (to appear). 
[WW1] M. Weiss and B. Williams, Automorphisms of manifolds and algebraic K-theory: I, K-Theory 1 (1988), 575-626.

[WW2] M. Weiss and B. Williams, Automorphisms of manifolds and algebraic K-theory: II, J. Pure and Appl. Algebra 62 (1989), 47-107.

[WW3] M. Weiss and B. Williams, Automorphisms of manifolds and algebraic K-theory: Finale, Preprint, 1994, 62pp.

[WW4] M. Weiss and B. Williams, Assembly, these Proceedings.

[WW5] M. Weiss and B. Williams, Pro-excisive functors, these Proceedings.

[Wu $\quad \mathrm{F}$. Wu, The higher index theorem for manifolds with boundary, J. Funct. Anal. 103 (1992), 160-189.

[Ya1] M. Yamasaki, L-groups of crystallographic groups, Invent. Math. 88 (1987), $571-602$.

[Ya2] M. Yamasaki, L-groups of virtually polycyclic groups, Topology and Appl. 33 (1989), 223-233.

[Ya3] M. Yamasaki, Maps between orbifolds, Proc. Amer. Math. Soc. 109 (1990), 223-232; Erratum, ibid. 115 (1992), 875.

[Yo] M. Yoshida, Surgery obstruction of twisted products, Math. J. Okayama Univ. 24 (1982), 73-97.

[Yu1] G. Yu, K-theoretic indices of Dirac-type operators on complete manifolds and the Roe algebra, preprint, Univ. of Colorado.

[Yu2] G. Yu, Cyclic cohomology and higher indices for noncompact complete manifolds, J. Funct. Anal. (to appear).

[Yu3] G. Yu, Baum-Connes conjecture and coarse geometry, K-Theory (to appear).

[Yu4] G. Yu, Localization algebra and coarse Baum-Connes conjecture, preprint, Univ. of Colorado.

[Yu5] G. Yu, Zero-in-the-spectrum conjecture, positive scalar curvature and asymptotic dimension, preprint, Univ. of Colorado.

Department of Mathematical Sciences, SUny at Binghamton, Binghamton, NY 13901, U.S.A.

email: steve@math.binghamton.edu

Department of Mathematics and Statistics, James Clerk Maxwell Bldg., University of Edinburgh, Edinburgh EH9 3JZ, Scotland, U.K.

email: a.ranicki@edinburgh.ac.uk

Department of Mathematics, University of Maryland, College Park, MD 20742, U.S.A.

email : jmr@math.umd.edu 